\title{
Multi-Task Generative Adversarial Network for Detecting Small Objects in the Wild
}

\author{
Yongqiang Zhang ${ }^{\dagger} \cdot$ Yancheng Bai ${ }^{\dagger} \cdot$ Mingli Ding * $\cdot$ Bernard Ghanem
}

Received: 23 December 2018 / Accepted: date

\begin{abstract}
Object detection results have been rapidly improved over a short period of time with the development of deep convolutional neural networks (DCNNs). Although impressive results have been achieved on large/medium sized objects, the performance on small objects is far from satisfactory and one of remaining open challenges is detecting small object in unconstrained conditions (e.g. COCO and WIDER FACE benchmarks). The reason is that small objects usually lack sufficient detailed appearance information, which can distinguish them from the backgrounds or similar objects. To deal with the small object detection problem, in this paper, we propose an end-to-end multi-task generative adversarial network (MTGAN), which is a general framework. In the MTGAN, the generator is a super-resolution network, which can up-sample small blurred images into fine-scale ones and recover detailed information for more accurate detection. The discriminator is a multitask network, which describes each inputted image patch with a real/fake score, object category scores, and bound-
\end{abstract}

$\dagger$ Equal contribution.

Yongqiang Zhang

School of Instrumentation Science and Engineering, Harbin Institute of Technology, Harbin, China.

E-mail: zhangyongqiang@hit.edu.cn

Yancheng Bai

Institute of Software, Chinese Academy of Sciences, Beijing, China.

E-mail: yancheng.bai.1987@gmail.com

Mingli Ding (* Corresponding author)

School of Instrumentation Science and Engineering, Harbin

Institute of Technology, Harbin, China.

E-mail: dingml@hit.edu.cn

Bernard Ghanem

Visual Computing Center, King Abdullah University of Science and Technology, Thuwal, Saudi Arabia.

E-mail: Bernardghanem@gmail.com ing box regression offsets. Furthermore, to make the generator recover more details for easier detection, the classification and regression losses in the discriminator are back-propagated into the generator during training process. Extensive experiments on the challenging COCO and WIDER FACE datasets demonstrate the effectiveness of the proposed method in restoring a clear super-resolved image from a blurred small one, and show that the detection performance, especially for small sized objects, improves over state-of-the-art methods by a large margin.

Keywords Small Object Detection - Small Face Detection · Super-resolution Network · Multi-task Generative Adversarial Network · COCO · WIDER FACE

\section{Introduction}

Object detection is a fundamental and important problem in computer vision. It is usually a key step towards many real-world applications, including image retrieval, intelligent surveillance, autonomous driving, etc. Object detection has been extensively studied over the past few decades and huge progress has been made with the emergence of deep convolutional neural networks (DCNNs). Currently, there are two main frameworks for CNN-based object detection: (i) the one-stage framework, such as YOLO (Redmon et al., 2016a) and SSD (Liu et al., 2016), which applies an object classifier and a location regressor in a dense manner without objectness pruning; and (ii) the two-stage framework, such as Faster-RCNN (Ren et al., 2015), RFCN (Dai et al., 2016) and FPN (Lin et al., 2017), which first extracts object proposals followed by per-proposal classification and regression. 


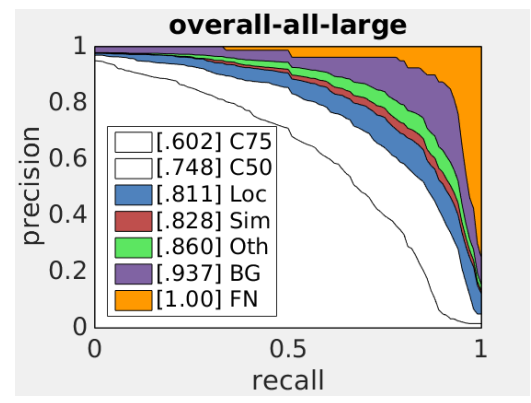

(a) Large

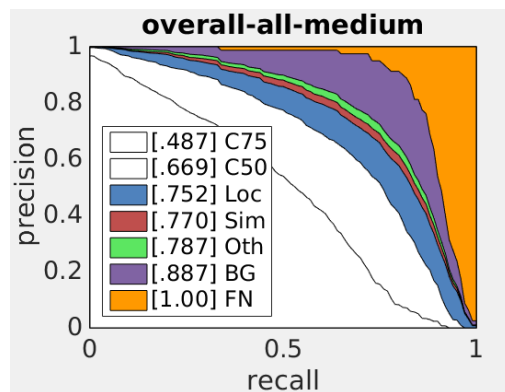

(b) Medium

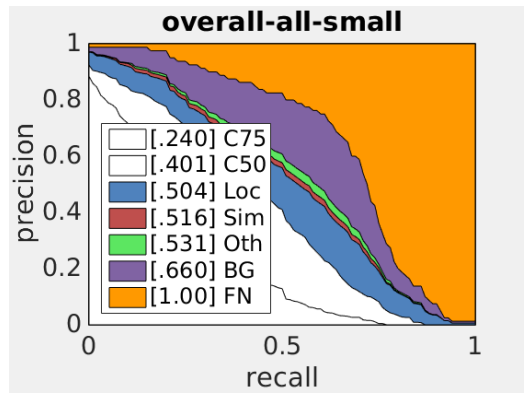

(c) Small

Fig. 1 The overall error analysis on the performance of the FPN detector (Lin et al., 2017) over all categories on the large, medium, and small subsets of the COCO dataset (Lin et al., 2014), respectively. The plots in each sub-image are a series of precision-recall curves under different evaluation settings defined in (Lin et al., 2014). The definition of legends in each sub-image can be found on the official website of COCO dataset ${ }^{2}$. From the comparisons, we can see that there is a large gap between the performance of small and large/medium sized objects.

Object detectors of both of the two frameworks have achieved impressive results on the objects of large/medium size in large-scale detection benchmarks (e.g. the COCO dataset (Lin et al., 2014)) as shown in Figure 1(a) and 1(b). However, the performance on small sized objects (defined as in (Lin et al., 2014)) is far from satisfactory as shown in Figure 1(c). From the comparisons, we can see that there is a large gap between the performance of small and large/medium sized objects. The main difficulty for small object detection (SOD) is that small objects lack appearance information, which is needed to distinguish them from background (or similar categories) and to achieve better localization. To achieve better detection performance on these small objects, some methods have been proposed in recent years. For example, SSD (Liu et al., 2016) exploits the intermediate conv feature maps to represent small objects. However, the shallow fine-grained conv feature maps are less discriminative, which leads to many false positive results. On the other hand, FPN (Lin et al., 2017) uses the feature pyramid to represent objects at different scales, in which low-resolution feature maps with strong semantic information are up-sampled and fused with the high-resolution feature maps with weak semantic information. However, direct up-sampling operation might generate artifacts, which may degrade detection performance.

To deal with the small object detection problem, we propose a unified end-to-end convolutional neural network based on the classical generative adversarial network (GAN) framework, which can be incorporated into any existing object detector. Following the structure of the seminal GAN work (Goodfellow et al., 2014; Ledig et al., 2017), there are two sub-networks in our proposed framework: a generator network and a dis-

2 http://cocodataset.org/\#detection-eval criminator network. In the generator network, a superresolution network (SRN) is introduced to up-sample a small object image to a larger scale one. Compared to directly re-sizing the image with bi-linear interpolation, SRN can generate images of higher quality and less artifacts at large up-scaling factors $(4 \times$ in our current implementation). In the discriminator network, we introduce the classification and regression branches for the task of object detection. The real natural highresolution and fake generated super-resolution images pass through the discriminator network that jointly distinguishes whether they are real natural high-resolution or fake generated super-resolution images, determines which classes they belong to, and refines the predicted bounding boxes. In Section 4, the ablation study experiments prove that adding regression branch is important for achieving superior performance, especially for the small cases. More importantly, the classification and regression losses are further back propagated to the generator network, which encourages the generator to produce higher quality images for easier classification and better localization.

Following the optimization strategy in classic generative adversarial network (Goodfellow et al., 2014), the generator network and discriminator network are optimized in an alternating manner and trained in an end-to-end way. The proposed loss functions will be presented in Section 3.

Contributions. This paper makes the following four main contributions. (1) A novel unified end-to-end multitask generative adversarial network (MTGAN) for small object detection is proposed, which can be incorporated with any existing detector. (2) In the MTGAN, the generator network produces super-resolved images and the multi-task discriminator network is introduced to distinguish the real natural high-resolution images 
from fake generated super-resolution ones, to predict object categories, and to regress bounding-boxes simultaneously. More importantly, the classification and regression losses are back-propagated to further guide the generator network to produce high-quality superresolution images for easier classification and better localization. (3) We validate the proposed MTGAN within the object detection pipeline on two popular challenging benchmarks (i.e. COCO (Lin et al., 2014) and WIDER FACE (Yang et al., 2016)), where detection performance improves a lot over several previous state-of-the-art baseline detectors, primarily for the small objects. (4) Finally, the proposed MTGAN presents good generalization ability across different datasets, and more experimental analysis are conducted to further demonstrate the effectiveness of the proposed MTGAN framework.

This paper is an extension version of our preliminary works accepted by CVPR18 (Bai et al., 2018a) and ECCV18 (Bai et al., 2018b). The major new contributions are that we change the architecture of the proposed networks in (Bai et al., 2018a), and additional experiments on the face detection dataset are conducted to demonstrate the generalization ability of the proposed method in (Bai et al., 2018b). Moreover, more experimental analysis are provided to verify the effectiveness of our proposed method. In summary, main changes contained in this paper are described as follows:

- Compare with (Bai et al., 2018a), we change the backbone network of the discriminator from VGG16 to ResNet50, which can learn stronger features for the inputted high-resolution/super-resolved patches. Moreover, to further refine the locations of the RoIs generated from the baseline detector, we add a regression branch in the discriminative network, which is parallel with the adversarial branch and classification branch. Meanwhile, the regression loss is further back-propagated to the loss function of the generator network to recover fine details for those superresolved images.

- Compare with (Bai et al., 2018b), we evaluate the proposed MTGAN framework for face detection on one of the most challenge face detection benchmark, i.e. WIDER FACE (Yang et al., 2016). We train the networks on the standard official training subset and evaluate the achieved model on the validation and test subsets. New state-of-the-art performance is reported when comparing with the contemporaneous face detectors, which demonstrates the effectiveness and the generalization ability of the proposed method.
- Finally, to further demonstrate the effectiveness of the proposed MTGAN framework, additional analysis and extensive experimental validation are included in this journal version. For instance, we replace the proposed super-resolution (i.e. generator) network with a simple bi-linear up-sampling method and further compare our proposed MTGAN with the plain R$\mathrm{CNN}$, integrate the results from different stages to verify if it can further improve the final performance, and further analyze the error types of the achieved performance.

The rest of the paper is organized as follows. We review the related work in Section 2. In Section 3, the detailed architecture of our GAN based small object detection framework and the objective function of the proposed framework are described. In Section 4, we present some experiments and ablation studies on two benchmarks (i.e. COCO and WIDER FACE), which contain a large number of small objects and are widely used recently in the task of object detection.Finally, the conclusions are provided in Section 5.

\section{Related Work}

\subsection{General Object \& Small-Object Detection}

As a classic topic, numerous object detection systems have been proposed during the past decade or so. Traditional object detection methods are based on handcrafted features and the deformable part model (DPM). Due to the limited representation of handcrafted features, traditional object detectors register subpar performance, particularly on small sized objects.

In recent years, superior performance in image classification and scene recognition has been achieved with the resurgence of deep convolutional neural networks (DCNNs) (Krizhevsky et al., 2012a; Simonyan and Zisserman, 2014; Zhou et al., 2014). Similarly, the performance of object detection (Cai and Vasconcelos, 2018; Bai et al., 2018b; Zhang et al., 2018b,c, 2019d) has been significantly boosted due to richer appearance and spatial representations, which are learned by CNNs (Girshick et al., 2014) from large scale image datasets. In general, a CNN-based object detector can be simply categorized as belonging to one of two frameworks: the two stage framework and the one stage framework.

The region-based CNN (RCNN) (Girshick et al., 2014) can be considered as a milestone of the two stage framework for object detection and it has achieved stateof-the-art detection performance. In RCNN, each region proposal is processed separately, which is very timeconsuming. After that, ROI-Pooling is introduced in 
Fast-RCNN (Girshick, 2015), which can share the computation between the proposal extraction and classification steps, thus improving the efficiency greatly. By learning both of these stages end-to-end, Faster RCNN (Ren et al., 2015) has registered further improvement in both detection performance and computational efficiency. However, all detectors of this framework show unsatisfactory performance on small sized objects in the COCO benchmark, since they do not have any explicit strategy to deal with such small objects. To detect small objects better, FPN (Lin et al., 2017) combines the semantically strong low-resolution features with semantically weak high-resolution features via a top-down pathway and lateral connections, in which the learned conv feature maps are expected to contain strong semantic information for small objects. Because of this, FPN shows superior performance over Faster RCNN for the task of detecting small objects. However, the low-resolution feature maps in FPN are up-sampled to create the feature pyramid, a process which tends to introduce artifacts to the features and consequently degrades the detection performance. Compared to FPN, our proposed method employs the super-resolution network to generate images with high-resolution $(4 \times$ upscaling) from images with low-resolution, thus, avoiding the artifact problem caused by the up-sampling operator in FPN.

In the one stage framework (Redmon et al., 2016b; Redmon and Farhadi, 2017a; Fu et al., 2017), the detector directly classifies anchors into specific classes and regresses bounding boxes in a dense manner. For example, in SSD (Liu et al., 2016) (a typical one-stage detector), the low-level intermediate conv feature maps of high-resolution are used to detect small objects. However, these conv features usually only capture basic visual patterns void of strong semantic information, which may lead to many false positive results. Compared to SSD-like detectors, our discriminator uses deep strong semantic features to better represent small objects, thus, reducing the false positive rate.

\subsection{Face \& Small-face Detection}

Existing face detectors can also be broadly divided into two categories: handcrafted feature based methods (Viola and Jones, 2004; Yan et al., 2014, 2013; Zhang et al., 2017c) and CNN-based methods (Cai et al., 2016; Jiang and Learned-Miller, 2017; Wan et al., 2016; Zhu et al., 2017a; Bai and Ghanem, 2017; Bai et al., 2018a; Wang et al., 2017a; Li et al., 2018; Wen et al., 2019). For convenience of comparison with our methods, in this paper, we just review the CNN-based methods.
Driven by the great success of RCNN, some works (Jiang and Learned-Miller, 2017; Wan et al., 2016; Zhu et al., 2017a; Tang et al., 2018; Chi et al., 2018; Zhang et al., 2017a, 2019b) employ this framework to detect faces, and impressive performance have been achieved on the simple benchmarks (e.g. FDDB (Jain and LearnedMiller, 2010)). However, performance drops dramatically on the more challenging benchmarks (e.g. WIDER FACE (Yang et al., 2016)), which contain lots of small faces. To overcome the problem of detecting small faces, traditional methods (Jiang and Learned-Miller, 2017; Wan et al., 2016; Zhu et al., 2017a) re-size input images to different scales during training and testing, which inevitably increases the GPU memory and computation costs. Furthermore, the re-size method often generates the images with large structural distortions when the target faces are too small. In general, detecting small objects requires fine scale representation, and some methods (Bai and Ghanem, 2017; Bell et al., 2016a) with skip connections have been proposed for this purpose, which combines fine-grained conv feature maps from shallow layers with coarse semantic features from deep layers to represent objects more precisely. However, simply incorporating all feature maps from different layers may not yield performance improvement. In fact, using these skip connections might decrease detection performance in some cases.

Another common method for small objects detection is using the contextual information to find these small cases. For instance, CMS-RCNN (Zhu et al., 2017a) imitates human vision system and uses explicit body contextual information in the proposed network for detecting the small faces. (Hu and Ramanan, 2017a) explores scale in-variance, image resolution and contextual reasoning in the task of detecting tiny faces, and shows that contextual information is crucial for detecting small and blurring faces in the wild. SSH (Najibi et al., 2017) proposes a simple context module to increase the effective receptive field on the conv layers for detecting the small targets. (Tang et al., 2018) introduces an context-assisted anchor-based method to utilize supervised information on learning contextual features for detecting small, blurred and partially occluded faces in the unconstrained conditions. However, using the contextual information may introduce some false positive results (e.g. a nose may be recognized as a face) and will increase the computational cost.

Compared to these methods, our proposed method first exploits the generator network to generate clear and fine faces with high resolution ( $4 \times$ up-scaling), and then the discriminator network is trained to distinguish the target faces from the input images. 


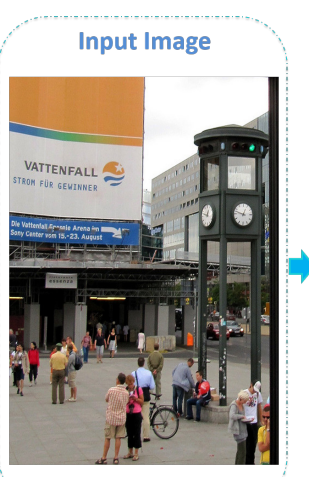

(A)

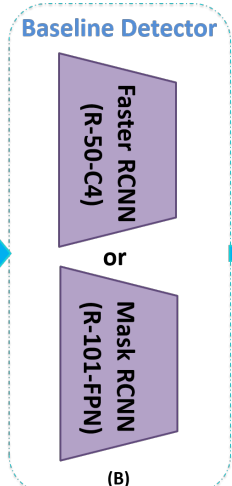

(B)

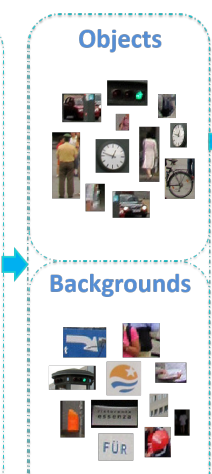

(c)
(D) Generator Network
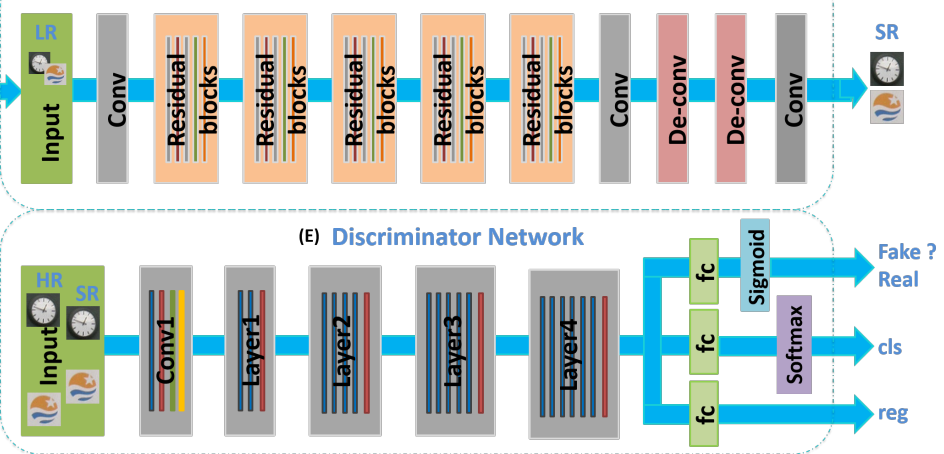

Fig. 2 The pipeline of the proposed multi-task generative adversarial network (MTGAN) for small objects detection. (A) The images are fed into the network. (B) The baseline detector can be any type of detector (e.g. Faster RCNN (Ren et al., 2015), FPN (Lin et al., 2017), or SSD (Liu et al., 2016)). It is used to crop positive (i.e. objects) and negative (i.e. background) examples from input images for training the generator and discriminator networks, or generate regions of interest (ROIs) for testing. (C) The positive and negative examples (or ROIs) are generated by off-the-shelf detectors. (D) The generator network reconstructs a super-resolved version ( $4 \times$ up-scaling) from the low-resolution input image. (E) The discriminator network distinguishes the real natural high-resolution from the fake generated super-resolution images, predicts the object categories, and regresses the object locations, simultaneously. Note that the discriminator network can use any typical architecture like AlexNet (Krizhevsky et al., 2012b), VGGNet (Simonyan and Zisserman, 2014) or ResNet (He et al., 2016) as the backbone network, and we use ResNet-50/ResNet-101 in our experiments.

\subsection{Generative Adversarial Networks}

In the seminal work (Goodfellow et al., 2014), the generative adversarial network (GAN) is introduced to generate realistic-looking images from random noise inputs. GANs have achieved impressive results in image generation (Denton et al., 2015), image editing (Zhu et al., 2016; Zhang et al., 2019a), representation learning (Mathieu et al., 2016), image super-resolution (Ledig et al., 2017; Song et al., 2019) and style transfer (Isola et al., 2017; Shiri et al., 2019). Recently, GANs have been successfully applied to super-resolution (SRGAN) (Ledig et al., 2017), leading to impressive and promising results. But SRGAN assumes that the input is a high-resolution image and contains fine details which are not available for the small objects in the wild. Compared to super-resolution on natural images, images of specific objects in the COCO or WIDER FACE benchmarks are full of diversity (e.g. blur, pose and illumination), thus, making the super-resolution process on these images much more challenging. In fact, the superresolution images generated by SRGAN are blurred especially for low-resolution small objects, which is not helpful to train an accurate object classifier.

To alleviate the above mentioned problem, we introduce novel losses into the loss function of the generator network, i.e. the classification and regression losses are back-propagated to the generator network in our proposed MTGAN, which further guides the generator to reconstruct finer super-resolved images for easier classification and better localization. In the discriminator network, the basic GANs are trained to distinguish the fake and real images, while we extend classic GAN to classify the fake vs. real images, recognize object $v s$. non-object, and regress location simultaneity by adding the classification and regression branches.

\section{MTGAN for Small Object Detection}

In this section, we introduce the proposed method in details. First, we give a brief description of the classical GAN network to lay the context for describing our proposed Multi-Task GAN (MTGAN) for small object detection. Then, the whole architecture of our framework is described (refer to Figure 2 for an illustration). Finally, we present each part of our MTGAN network and define the loss functions for training the generator and discriminator networks, respectively.

\subsection{Revisiting Generative Adversarial Network (GAN)}

GAN (Goodfellow et al., 2014) learns a generator network $G$ and a discriminator network $D$ simultaneously via an adversarial process. The training process alternately optimizes the generator and discriminator networks, which are in competition with each other. The generator $G$ is trained to produce samples to fool the discriminator $D$, and $D$ is trained to distinguish real 
Table 1 The architecture of the generator and discriminator networks. "conv" and "layer*" represent the convolutional layer, "x5" denotes a residual block which has 5 convolutional layers, "de-conv" means a up-sampling convolutional layer, "2x" denotes up-sampling by a factor of 2 , and "fc" indicates the fully connected layer. Note that we only post the architecture of the discriminator network with ResNet-50.

\begin{tabular}{|c|c|c|c|c|c|c|c|c|c|c|c|c|}
\hline & \multicolumn{6}{|c|}{ Generator } & \multicolumn{6}{|c|}{ Discriminator (ResNet-50) } \\
\hline Layer & conv & conv $\times 5$ & conv & de-conv & de-conv & conv & conv & layer1 & layer2 & layer3 & layer4 & $\mathrm{fc}$ \\
\hline Kernel Num. & 64 & 64 & 64 & 256 & 256 & 3 & 64 & 128 & 256 & 512 & 1024 & 3 \\
\hline Kernel Size & 9 & 3 & 3 & 3 & 3 & 9 & 3 & 1 & 1 & 1 & 1 & - \\
\hline
\end{tabular}

images from fake ones produced by $G$. The classical GAN loss to be optimized is defined as follows:

$$
\begin{aligned}
\mathcal{L}_{G A N}(G, D)= & \mathbb{E}_{x \sim p_{\text {data }}(x)}\left[\log D_{\theta}(x)\right]+ \\
& \mathbb{E}_{z \sim p_{z}(z)}\left[\log \left(1-D_{\theta}\left(G_{\omega}(z)\right)\right)\right],
\end{aligned}
$$

where $z$ is random noise, $x$ denotes the real data, $\theta$ and $\omega$ denote the parameters of $D$ and $G$ respectively. Here, generator $G$ tries to minimize the objective function, while discriminator $D$ tries to maximize it as $\mathrm{Eq}(2)$ :

$\arg \min _{G} \max _{D} \mathcal{L}_{G A N}(G, D)$.

Similar to classical GANs (Goodfellow et al., 2014; Ledig et al., 2017), we design a generator network $G_{w}$ which is optimized in an alternating manner with discriminator network $D_{\theta}$, seeking to jointly solve the superresolution, object classification, and bounding box regression problems for small object detection. Therefore, the overall loss is defined as follows:

$$
\begin{aligned}
\arg \min _{G_{w}} \max _{D_{\theta}} \mathcal{L}_{G A N}\left(G_{w}, D_{\theta}\right)+ \\
L_{M S E}(w)+L_{c l s}(u, \theta)+L_{r e g}(v, \theta),
\end{aligned}
$$

where $\mathcal{L}_{G A N}\left(G_{w}, D_{\theta}\right)$ represents the adversarial loss, $L_{M S E}(w)$ denotes pixel-wise loss (super-resolution loss), $L_{c l s}(u, \theta)$ means the classification loss and $L_{r e g}(v, \theta)$ denotes the regression loss, which are defined in subsection 3.3. $w$ and $\theta$ denote parameters of generator and discriminator networks, respectively. $u$ is the class label and $v$ is the ground-truth bounding-box regression target. Unlike (Goodfellow et al., 2014), the input of our generator is a low-resolution image $I^{L R}$ rather than random noise $z$. Compared to (Ledig et al., 2017), we have multiple tasks in the discriminator network, where we distinguish the fake generated super-resolved images vs. real natural high-resolution images, classify the object category, and regress the object location jointly. Specifically, the general idea behind Eq (3) is that it allows one to train a generator $G$ with the goal of fooling a differentiable discriminator $D$ that is trained to distinguish fake super-resolved images from real highresolution images. Furthermore, our proposed method (MTGAN) extends classical SRGAN (Ledig et al., 2017) by adding two more parallel branches to classify the categories and regress the bounding boxes of candidate ROI images. Moreover, the classification and regression losses in the discriminator are back-propagated to the generator network to further promote it to produce super-resolved images with rich details that are also suitable for easier classification and better localization. In the following subsection, we introduce the architecture of the proposed MTGAN and the training losses in details.

\subsection{Network Architecture}

Our generator network takes low-resolution images as input, instead of random noise, and outputs super-resolved images. For the purpose of object detection, the discriminator network is designed to distinguish fake generated super-resolved images from real high-resolution images, classify the object categories, and regress the location jointly.

Generator Network $\left(G_{w}\right)$. As shown in Table 1 and Figure 2, we adopt a deep CNN architecture which has shown effectiveness for image de-blurring in (Hradiš et al., 2015), general object detection in (Bai et al., 2018b) and face detection in (Bai et al., 2018a). Different from (Hradiš et al., 2015), our generator network includes up-sampling layers (i.e. de-conv in Table 1). There are two up-sampling fractionally-strided conv layers, three conv layers, and five residual blocks in the generator network. Particularly, in these residual blocks, we use two conv layers with $3 \times 3$ kernels and 64 feature maps followed by batch-normalization layers (Ioffe and Szegedy, 2015) and parametric ReLU (He et al., 2015) as the activation function. Each deconvolutional layer consists of learned kernels, which up-samples a low-resolution image to a $2 \times$ super-resolved image, which is usually better than re-sizing the same image by an interpolation method (Dong et al., 2016; Kim et al., 2016; Wang et al., 2015).

Our generator first up-samples the low-resolution small images, which include both object and background candidate ROI images, to $4 \times$ super-resolved images via the de-convolutional layers, and then performs convolution to produce corresponding clear images. The out- 
puts of the generator network (i.e. clear super-resolved images) are easier for the discriminator to classify as fake generated super-resolved images vs. real natural high-resolution ones and to perform object detection (i.e. object classification and bounding-box regression), respectively.

Discriminator Network $\left(D_{\theta}\right)$. We employ ResNet50/ResNet-101 (He et al., 2016) as our backbone network in the discriminator network, and Table 1 and Figure 2 show the architecture of the ResNet-50 network. Different from the original ResNet architecture, we add three parallel $f c$ layers behind the last average pooling layer of the backbone network, which play the role of distinguishing the real natural high-resolution images from the fake generated super-resolved ones, classifying object categories, and regressing bounding boxes, respectively. For this specific task, the first $f c$ layer (called $f c_{G A N}$ ) uses a sigmoid loss function (Radford et al., 2015), while the classification $f c$ layer (called $f c_{c l s}$ ) and regression $f c$ layer (called $f c_{r e g}$ ) use the softmax and smooth $L 1$ loss (Girshick, 2015) functions after each $f c$ layer, respectively.

The input of the discriminator network is the highresolution ROI images, which include real high-resolution and fake generated super-resolved images. The output of the $f c_{G A N}$ branch, termed as adversarial branch, is the probability $\left(p_{G A N}\right)$ of the input image being a real image, the output of $f c_{c l s}$ branch (called classification branch $)$ is the probability $\left(p_{c l s}=\left(p_{0}, \ldots, p_{K}\right)\right)$ of the input image being each of $K+1$ object categories, and the output of $f c_{r e g}$ branch, coined as regression branch, is the bounding-box regression offsets $\left(t_{r e g}=\left(t_{x}, t_{y}, t_{w}, t_{h}\right)\right)$ for each inputted ROI candidate. Note that the final detection results are directly from the classification and regression branches and no subsequent operation to further process the achieved results.

\subsection{Overall Loss Function}

We adopt the pixel-wise and adversarial losses from the state-of-the-art GAN approaches (Ledig et al., 2017; Isola et al., 2017) to optimize our proposed networks. In contrast to (Ledig et al., 2017), we remove the feature matching loss to decrease the computational complexity without sacrificing much in generation performance. Furthermore, we introduce the classification and regression losses into the objective function to classify the object categories and regress the location in the discriminator network, and further to drive the generator network to recover fine details from small scale images for easier detection and better localization.

Pixel-wise Loss. The input of our generator network is small ROI images instead of the random noise in (Goodfellow et al., 2014). A natural and simple way to enforce the output of the generator network (i.e. the super-resolved images) to be close to the ground-truth images (i.e. the high-resolution images) is by minimizing the pixel-wise MSE loss, and it is computed as Eq (4):

$L_{M S E}(w)=\frac{1}{N} \sum_{i=1}^{N}\left\|G_{w}\left(I_{i}^{L R}\right)-I_{i}^{H R}\right\|^{2}$,

where $I_{i}^{L R}, G_{w}\left(I_{i}^{L R}\right)$ and $I_{i}^{H R}$ denote small low-resolution images, fake generated super-resolved images, and real natural high-resolution images, respectively. $G$ represents the generator network, and $w$ denotes its parameters. However, it is known that the solution to the MSE optimization problem usually lacks high-frequency content, which results in blurred images with overly smooth texture.

Adversarial Loss. Since super-resolution is inherently an under-determined problem, there are many possible mappings between low-resolution and superresolved images. So far, there is no standard loss function to guarantee that the super-resolution network can output realistic super-resolved images. To achieve more realistic results, we introduce the adversarial loss (Ledig et al., 2017) to the objective loss, defined as $\operatorname{Eq}(5)$ :

$L_{a d v}=\frac{1}{N} \sum_{i=1}^{N} \log \left(1-D_{\theta}\left(G_{w}\left(I_{i}^{L R}\right)\right)\right)$,

where $D$ represents the discriminator network, and $\theta$ denotes its parameters.

The adversarial loss guides the discriminator network to learn a classifier that classifies high-resolution images, albeit fake generated super-resolved and real high-resolution images, as real or fake. In addition, it also encourages the generator $G$ to produce sharper high-frequency details so as to fool the discriminator $D$. In Eq (5), $D_{\theta}\left(G_{w}\left(I_{i}^{L R}\right)\right)$ denotes the probability of the super-resolved image $G_{w}\left(I_{i}^{L R}\right)$ being a real highresolution image.

Classification Loss. In order to complete the task of object detection and to make the generated superresolved images easier to classify, we introduce the classification loss to the overall objective. Let $\left\{I_{i}^{L R}, i=1,2\right.$, $\ldots, N\}$ and $\left\{I_{i}^{H R}, i=1,2, \ldots, N\right\}$ denote low-resolution images and real high-resolution images respectively, and $\left\{u_{i}, i=1,2, \ldots, N\right\}$ represents their corresponding labels, where $u_{i} \in\{0, \ldots, K\}$ indicates the object category. As such, we formulate the classification loss as $\operatorname{Eq}(6)$ :

$$
\begin{gathered}
L_{c l s}(p, u)=\frac{1}{N} \sum_{i=1}^{N}-\left(\log \left(D_{c l s}\left(G_{w}\left(I_{i}^{L R}\right)\right)\right)+\right. \\
\left.\log \left(D_{c l s}\left(I_{i}^{H R}\right)\right)\right),
\end{gathered}
$$


where $p_{I_{i}^{L R}}=D_{c l s}\left(G_{w}\left(I_{i}^{L R}\right)\right)$ and $\left.p_{I_{i}^{H R}}=D_{c l s}\left(I_{i}^{H R}\right)\right)$ denote the probabilities of the fake generated superresolved and the real high-resolution images belonging to the true category $u_{i}$, respectively.

In our proposed method, our classification loss plays two roles. First, it guides the discriminator network to learn a classifier that classifies which category the input high-resolution and super-resolved ROI images belong to. Second, it promotes the generator network to recovery clearer images for easier classification and better localization.

Regression Loss. For small object detection, just a few pixel shift may lead to failure detection. To enable more accurate localization, we also introduce a bounding box regression loss (Girshick, 2015) to the objective function, defined in $\mathrm{Eq}(7)$ :

$$
\begin{array}{r}
L_{r e g}(t, v)=\frac{1}{N} \sum_{i=1}^{N} \sum_{j \in\{x, y, w, h\}}\left[u_{i} \geq 1\right]\left(\mathrm{S}_{L_{1}}\left(t_{i, j}^{H R}-v_{i, j}\right)+\right. \\
\left.\mathrm{S}_{L_{1}}\left(t_{i, j}^{S R}-v_{i, j}\right)\right)
\end{array}
$$

in which,

$\mathrm{S}_{L_{1}}(x)= \begin{cases}0.5 x^{2} & \text { if }|x|<1 \\ |x|-0.5 & \text { otherwise }\end{cases}$

where $v_{i}=\left(v_{i, x}, v_{i, y}, v_{i, w}, v_{i, h}\right)$ denotes a tuple of the true bounding-box regression target, and $t_{i}=\left(t_{i, x}, t_{i, y}\right.$, $\left.t_{i, w}, t_{i, h}\right)$ denotes the predicted regression tuple. $t_{i}^{H R}$ and $t_{i}^{S R}$ denote the tuples for the $i$-th real high-resolution and fake generated super-resolved images, respectively. The bracket indicator function $\left[u_{i} \geq 1\right]$ equals to 1 when $u_{i} \geq 1$ and 0 otherwise. For a more detailed description of the regression loss, we refer the reader to (Girshick, 2015).

Similar to the classification loss $\mathrm{Eq}(6)$, our regression loss also has two purposes. The first purpose is that it supervises the discriminator to regress the location of the object candidates cropped from the baseline detector. The second one is that it promotes the generator to produce super-resolved images with fine details for more accurate localization.

Objective Function. Based on the above analysis, we combine the adversarial loss in Eq (5), classification loss in Eq (6) and regression loss in Eq (7) with the pixel-wise MSE loss in Eq (4) to train the generator and discriminator networks. As such, our proposed MTGAN network can be trained by optimizing the ob- jective function in $\mathrm{Eq}(9)$ :

$$
\begin{aligned}
\max _{\theta} \min _{w} & \frac{1}{N} \sum_{i=1}^{N} \alpha\left(\log \left(1-D_{\theta}\left(G_{w}\left(I_{i}^{L R}\right)\right)\right)+\log D_{\theta}\left(I_{i}^{H R}\right)\right)+ \\
& \frac{1}{N} \sum_{i=1}^{N}-\beta\left(\log \left(D_{c l s}\left(G_{w}\left(I_{i}^{L R}\right)\right)\right)+\log \left(D_{c l s}\left(I_{i}^{H R}\right)\right)\right)+ \\
& \frac{1}{N} \sum_{i=1}^{N} \sum_{j \in\{x, y, w, h\}} \gamma\left[u_{i} \geq 1\right]\left(\mathrm{S}_{L_{1}}\left(t_{i, j}^{H R}-v_{i, j}\right)+\right. \\
& \frac{1}{N} \sum_{i=1}^{N}\left\|G_{w}\left(I_{i}^{L R}\right)-I_{i}^{H R}\right\|^{2},
\end{aligned}
$$

where $\alpha, \beta$, and $\gamma$ are weights trading off the different terms, which are set through the cross-validation experiments.

However, directly optimizing Eq (9) with respect to $w$ for updating generator $G$ makes $w$ diverge to infinity rapidly, since large $w$ always makes the objective attain a large loss. For better behavior, we optimize the objective function in a fixed point optimization manner, as done in previous GAN works (Ledig et al., 2017; Isola et al., 2017). Specifically, we optimize the parameter $w$ of generator $G$ while keeping the parameter $\theta$ of discriminator $D$ fixed, and then update the parameter $\theta$ keeping the parameter $w$ fixed. Below are the two resulting sub-problems that are iteratively optimized as $\mathrm{Eq}(10)$ and $\mathrm{Eq}(11)$ :

$$
\begin{aligned}
\min _{w} & \frac{1}{N} \sum_{i=1}^{N} \alpha \log \left(1-D_{\theta}\left(G_{w}\left(I_{i}^{L R}\right)\right)\right)- \\
& \frac{1}{N} \sum_{i=1}^{N} \beta \log \left(D_{c l s}\left(G_{w}\left(I_{i}^{L R}\right)\right)\right)+ \\
& \frac{1}{N} \sum_{i=1}^{N} \sum_{j \in\{x, y, w, h\}} \gamma\left[u_{i} \geq 1\right] \mathrm{S}_{L_{1}}\left(t_{i, j}^{S R}-v_{i, j}\right)+ \\
& \frac{1}{N} \sum_{i=1}^{N}\left\|G_{w 1}\left(I_{i}^{L R}\right)-I_{i}^{H R}\right\|^{2}
\end{aligned}
$$

and

$$
\begin{aligned}
& \min _{\theta} \frac{1}{N} \sum_{i=1}^{N}-\alpha\left(\log \left(1-D_{\theta}\left(G_{w}\left(I_{i}^{L R}\right)\right)\right)+\log D_{\theta}\left(I_{i}^{H R}\right)\right)+ \\
& \frac{1}{N} \sum_{i=1}^{N}-\beta\left(\log \left(D_{c l s}\left(G_{w}\left(I_{i}^{L R}\right)\right)\right)+\log \left(D_{c l s}\left(I_{i}^{H R}\right)\right)\right)+ \\
& \frac{1}{N} \sum_{i=1}^{N} \sum_{j \in\{x, y, w, h\}} \gamma\left[u_{i} \geq 1\right]\left(\mathrm{S}_{L_{1}}\left(t_{i, j}^{H R}-v_{i, j}\right)+\right. \\
&\left.\mathrm{S}_{L_{1}}\left(t_{i, j}^{S R}-v_{i, j}\right)\right) .
\end{aligned}
$$


The loss function of generator $G$ in $\mathrm{Eq}(10)$ consists of adversarial loss $\mathrm{Eq}(5)$, MSE loss $\mathrm{Eq}(4)$ and classification loss $\mathrm{Eq}(6)$ \& regression loss $\mathrm{Eq}(7)$, which enforce that the reconstructed super-resolved images to be similar to the real natural high-resolution image on the high-frequency details, pixel, and semantic level respectively. Compared to the previous GANs, we add the classification and regression losses of generated superresolved object images to the objective loss of the generator network. By introducing these two losses, the super-resolved images recovered from the generator network are more realistic than those optimized by only using the adversarial and MSE losses. Of course, the generated SR images from the designed generator are clearer (i.e. including more high-frequency details) than the re-seized images created by using the bi-linear interpolation method, and the corresponding ablation studies are shown in Section 4.3.

The loss function of discriminator $D$ in Eq (11) includes adversarial loss Eq(5), classification loss Eq (6) and regression loss Eq (7). The function of classification loss is to classify the categories of the real highresolution and fake generated super-resolved ROI images, which is parallel to the basic formulation of GAN (Goodfellow et al., 2014) to distinguish real or generated high-resolution images. In the field of small object detection, as we all know, a few pixel shift may make the predicted bounding-boxes fail to fulfill the evaluation criteria (i.e. IoU $>0.5$ in WIDER FACE dataset or IoU > [0.5:0.05:0.95] in COCO dataset between the predicted bounding-boxes and the ground-truth boundingboxes). Therefore, we introduce the regression loss (regression branch) into the discriminator network for better localization.

The ablation analysis revealing the influence of each component, including the adversarial, classification and regression losses in the generator network, and the regression branch in the discriminator network, are presented in Section 4.3.

\section{Experiments}

In this section, we experimentally validate our proposed MTGAN detector on two challenging public detection benchmarks (i.e. COCO dataset (Lin et al., 2014) and WIDER FACE dataset (Yang et al., 2016)). First, we conduct an ablation experiment to prove the effectiveness of the proposed MTGAN framework. Then, ablation studies are conducted to verify the positive influence of the regression branch and the effectiveness of each loss in the generator and discriminator networks respectively. Finally, our proposed method is evaluated on the COCO and WIDER FACE datasets with comparisons against other previous state-of-the-art detectors.

\subsection{Training and Validation Datasets}

We evaluate our proposed method on COCO dataset (Lin et al., 2014) and WIDER FACE dataset (Yang et al., 2016). These two datasets are the most widely used benchmarks in the object detection task now. More importantly, there are a large number of small objects in these two datasets, therefore we use them for training and validating our proposed MTGAN detector.

COCO dataset. As stated in (Lin et al., 2014), there are more small objects than large/medium objects in the dataset, approximately $41 \%$ of objects are small $\left(\right.$ area $\left.<32^{2}\right)$. For the object detection task, there are $125 \mathrm{~K}$ images taken in natural settings and from everyday life (i.e. objects with much diversity). $80 \mathrm{~K} / 40 \mathrm{~K} / 5 \mathrm{~K}$ of the images are randomly selected for training, validation, and testing, respectively. Following previous works (Bell et al., 2016b; Lin et al., 2017), we use the union of $80 \mathrm{k}$ training images and a subset of $35 \mathrm{k}$ validation images (trainval135k) for training, and report ablation results on the remaining $5 \mathrm{k}$ validation images (minival). During evaluation, the COCO dataset is divided into three subsets (small, medium and large) based on the areas of objects. The medium and large subsets contain objects with an area larger than $32^{2}$ and $96^{2}$ pixels, respectively, while the small subset contains objects with an area less than $32^{2}$ pixels. As expected, it is quite challenging to achieve good detection performance on the small subset. In this paper, we focus on small object detection using our proposed MTGAN framework. We report the final detection performance on the test subset by using the standard COCO metrics, which include AP (averaged over all IoU thresholds, i.e. [0.5:0.05:0.95]), $\mathrm{AP}_{50}, \mathrm{AP}_{75}$ and $\mathrm{AP}_{S}, \mathrm{AP}_{M}$, $\mathrm{AP}_{L}$ (AP at different scales).

WIDER FACE dataset. There are 32,203 images in the WIDER FACE dataset (Yang et al., 2016), which are picked from the WIDER dataset. Following the official setting in (Yang et al., 2016), 40\%/10\%/50\% of the images are randomly divided for training, validation, and testing, respectively. Images in the WIDER FACE dataset are categorized into 61 social event classes, which cover almost all diversity social scenarios and are closer to the real-world scenes. In the evaluation process, the WIDER FACE dataset is divided into three subsets ( Easy, Medium, and Hard) based on the heights of the annotated ground truth faces. The Easy/Medium/Hard subsets contain faces with heights larger than 50/30/10 pixels respectively. Compared to the Medium and Easy 
subsets, the Hard one contains faces with a height between 10-30 pixels. As all know is that it is also quite challenging to achieve good detection performance on this subset. In this paper, we focus on detecting these small faces and report the detection performance on the validation subset by using the standard AP metrics in (Yang et al., 2016).

\subsection{Implementation Details}

In the generator network, we set the trade-off weights $\alpha=0.001, \beta=\gamma=0.01$. The generator network is trained from scratch and the weights in each layer are initialized with a zero-mean Gaussian distribution with standard deviation 0.02 , and the biases are initialized with 0 . To avoid undesirable local optima, we first train an MSE-based super-resolution network to initialize the generator network. For the discriminator network, we employ the ResNet-50 or ResNet-101 (He et al., 2016) model pre-trained on ImageNet as our backbone network and add three parallel $f c$ layers as described in Section 3.2. The $f c$ layers are initialized by a zero-mean Gaussian distribution with standard deviation 0.1, and biases initialized with 0 .

In the training process, we use the Adam optimizer (Kingma and Ba, 2014) for optimizing the generator network and adopt the SGD optimizer for optimizing the discriminator network respectively. The generator and discriminator networks are alternately updated as in (Goodfellow et al., 2014). During testing, we follow the standard testing code as in (Girshick, 2015) and (Yang et al., 2016) to evaluate the achieved model. Our implementation is based on Pytorch, and all the experiments are done on an NVIDIA TITAN Xp GPU with $12 \mathrm{G}$ memory.

COCO dataset. The baseline detectors are based on Faster RCNN (Ren et al., 2015) with ResNet50C4 (He et al., 2016) and FPN (Lin et al., 2017) with ResNet101 (Lin et al., 2017). All hyper-parameters of the baseline detectors are adopted from the setups in (He et al., 2017). To train our generator and discriminator networks, we crop positive and negative ROI examples from COCO (Lin et al., 2014) trainval135k set with our baseline detectors. The corresponding lowresolution images are generated by down-sampling the high-resolution images using bicubic interpolation with a factor 4. The learning rate for SGD optimizer is initially set to 0.01 and then reduced by a factor of 10 after every $40 k$ mini-batches. Training is terminated after a maximum of $80 k$ iterations. During testing, 100 ROIs are cropped by our baseline detector and then fed to our MTGAN framework to produce the final detection performance.

WIDER FACE dataset. We choose SRFACE detector (Bai et al., 2018a) with VGG19 as our baseline detector, and all hyper-parameters of the baseline detector are the same as the original implementation in (Bai et al., 2018a). Similarly, we crop face and nonface samples from WIDER FACE training set based on our baseline detector to train the generator and discriminator networks, and the low-resolution images are achieved by down-sampling the corresponding highresolution images by using the bicubic interpolation with a factor 4 . The networks are trained with first 3 epochs at a learning rate of $10^{-4}$ and divided by a factor 10 in another 3 epochs. In the testing process, 600 regions of interest (ROIs) are cropped and further fed to our proposed MTGAN framework to give the final detection performance.

\subsection{Ablation Studies}

We first compare our proposed method with the baseline detectors and plain R-CNN (Girshick et al., 2014) to prove the effectiveness of the MTGAN framework for small object detection. Furthermore, we demonstrate the effectiveness of the proposed generator in the MTGAN framework by comparing with the up-sampling method. Moreover, we verify the positive influence of the regression branch in the discriminator network by comparing the AP performance with/without this branch. Then, to validate the contribution of each loss (adversarial, classification, and regression) in the loss function of the generator network, we also conduct ablation studies by gradually adding each of them to the pixel-wise MSE loss. Finally, we conduct an experiment to further validate the effectiveness of the proposed method through answering a question whether integrating results from two different stages can improve the final detection performance. Unless otherwise stated, all the ablation studies use the ResNet-50 as the backbone network in the discriminator and are conducted on the COCO dataset.

Compared MTGAN with baseline detectors. Table 2 (the $2^{\text {nd }}$ vs. $3^{\text {rd }}$ row and the $4^{\text {th }}$ vs. $5^{\text {th }}$ row) compares the performance of the baseline detectors against our method MTGAN on the COCO minival subset. In order to prove the effectiveness of our method, we choose two popular object detectors (i.e. Faster-RCNN and Mask-RCNN) with high performance as our base-

\footnotetext{
4 In other Tables, the item "Backbone" has the same meaning with Table 2, i.e. it means the backbone network of our discriminator unless otherwise stated.
} 
Table 2 The detection performance (AP) of our proposed method MTGAN against the baseline methods on the COCO minival subset. Note that the "Backbone" ${ }^{4}$ in our proposed MTGAN framework means the backbone network of discriminator. The AP performance of Faster RCNN (Ren et al., 2015) and Mask-RCNN (He et al., 2017) are provided by (Girshick et al., 2018). Obviously, MTGAN outperforms the baseline methods, especially on the small subset where the AP performance increases by more than $1.5 \%$.

\begin{tabular}{r|l|lll|lll}
\hline Methods & Backbone & $\mathrm{AP}$ & $\mathrm{AP}_{50}$ & $\mathrm{AP}_{75}$ & $\mathrm{AP}_{S}$ & $\mathrm{AP}_{M}$ & $\mathrm{AP}_{L}$ \\
\hline \hline Faster-RCNN (Baseline) & ResNet-50-C4 & 36.5 & 57.3 & 39.3 & 18.4 & 40.6 & 50.6 \\
MTGAN (Ours) & ResNet-50 & $\mathbf{3 7 . 2}$ & $\mathbf{5 7 . 7}$ & $\mathbf{4 0 . 2}$ & $\mathbf{1 9 . 9}$ & $\mathbf{4 1 . 2}$ & $\mathbf{5 1 . 2}$ \\
\hline \hline Mask-RCNN (Baseline) & ResNet-101-FPN & 40.9 & 61.9 & 44.8 & 23.5 & 44.2 & 53.9 \\
MTGAN (Ours) & ResNet-101 & $\mathbf{4 1 . 5}$ & $\mathbf{6 2 . 5}$ & $\mathbf{4 5 . 4}$ & $\mathbf{2 5 . 1}$ & $\mathbf{4 4 . 6}$ & $\mathbf{5 4 . 1}$ \\
\hline
\end{tabular}

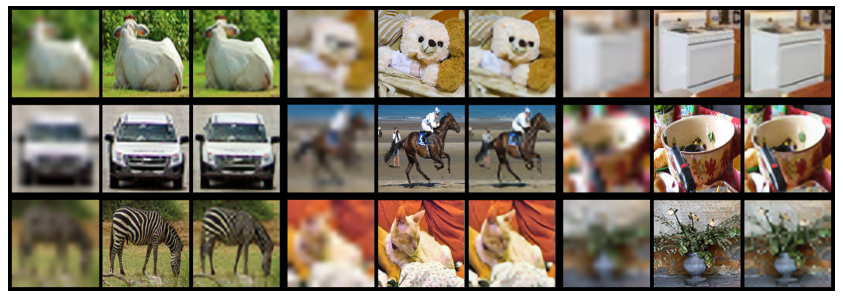

Fig. 3 Some examples of super-resolved images generated by the generator in our proposed MTGAN framework from small low-resolution patches. The first column of each image group depicts the original low-resolution image, which is up-sampled $4 \times$ for visualization. The second column is the ground truth high-resolution image, while the third column is the corresponding super-resolved image generated by our generator network.

line, and for more detailed information about the baseline detectors please refer to (Ren et al., 2015) and (He et al., 2017) respectively. We would like to stress that the baseline detector is not specific and any off-the-shelf detector can be integrated into our framework. From Table 2, we observe that the performance of our MTGAN with ResNet-50 outperforms Faster-RCNN with ResNet-50-C4 by a sizable margin (i.e. $1.5 \%$ in AP) on the small subset. Similarly, MTGAN with ResNet-101 improves over the FPN detector with ResNet-101 by $1.6 \%$ in AP performance. The reason is that the baseline detectors perform the down-sampling operations (i.e. convolution with stride 2) when extracting conv feature maps. The small objects themselves contain limited information, and the majority of the detailed information will be lost after several down-sampling operations. For example, if the input is a $16 \times 16$ pixels object ROI, the result is a $1 \times 1 \mathrm{C} 4$ feature map and nothing is preserved in the C5 feature maps. These limited conv feature maps degrade the detection performance for such small objects. In contrast, our method up-samples the low-resolution image to a fine scale, thus, recovering the detailed information and making detection possible. Based on the clear super-resolution images with fine details, the boosting of the detection performance is inevitable.
Figure 3 shows some super-resolved images generated by the generator in our proposed MTGAN framework. We observe that our method can generate the clear super-resolution images from the small ones, which is better for detection. For some really small blurred cases, even though the generated images lack fine high frequency details, but a clear contour is recovered, which is enough for classification and regression in the task of detection.

Compared MTGAN with Plain R-CNN. Actually, as done in plain R-CNN (Girshick et al., 2014), directly re-sizing the input image to a canonical dimension (e.g. $224 \times 224)$ with bi-linear interpolation method might generate artifacts and the generated images are usually blurred for the small input patches (e.g. 16×16). In contrast, our proposed super-resolution network can generate images of higher quality and less artifacts at large up-scaling factors (e.g. $4 \times$ ). As shown in Figure 3, the first column of each image group depicts the directly re-sized image, which is blurred and some of them even can not be recognized as an objects for the small complicated objects, and the third column is the corresponding super-resolved image generated by our generator network.

To validate our method is more effective than plain R-CNN on detecting small objects, we conduct an experiment by replacing the proposed super-resolution generator and discriminator with a plain R-CNN. As the original architecture of R-CNN in (Girshick et al., 2014), we first re-size the positive and negative examples (i.e. proposals) generated by our baseline detector to a standard scale (i.e. $224 \times 224$ ), and then employ ResNet50 to learn features of the input patches. Finally, class-specific linear SVMs are used to perform classification task. As shown in Table 3 ( $1^{\text {st }}$ vs. $3^{\text {rd }}$ rows), the performance of the plain R-CNN is far behind the performance of our proposed method. There are three reasons for this unsatisfactory performance: 1) the classification head and the feature learning network are not trained in an end-to-end manner, 2) the classification ability of the linear SVM is weak, 3) no regression branch to further refine the location of each pro- 
Table 3 The performance of our proposed method (MTGAN) against the plain R-CNN and our discriminator network without adversarial branch on the COCO minival subset. "D" denotes the discriminator network in our proposed MTGAN framework, and "adv+" indicates the adversarial branch, which distinguishes the generated SR images from the real HR images, in the discriminator network

\begin{tabular}{r|lll|lll}
\hline Methods & $\mathrm{AP}$ & $\mathrm{AP}_{50}$ & $\mathrm{AP}_{75}$ & $\mathrm{AP}_{S}$ & $\mathrm{AP}_{M}$ & $\mathrm{AP}_{L}$ \\
\hline \hline Plain R-CNN & 21.8 & 40.6 & 19.6 & 10.6 & 20.3 & 32.6 \\
D w/o adv+ branch & 36.1 & 57.1 & 39.1 & 18.8 & 40.3 & 49.4 \\
MTGAN (Ours) & 37.2 & 57.7 & 40.2 & 19.9 & 41.2 & 51.2 \\
\hline
\end{tabular}

Table 4 Performance of the model trained with the SBU and MTGAN, where "SBU" denotes the method of using a simple bi-linear up-sampler to generated super-resolved images.

\begin{tabular}{r|cll|lll}
\hline Methods & $\mathrm{AP}$ & $\mathrm{AP}_{50}$ & $\mathrm{AP}_{75}$ & $\mathrm{AP}_{S}$ & $\mathrm{AP}_{M}$ & $\mathrm{AP}_{L}$ \\
\hline \hline Baseline & 36.5 & 57.3 & 39.3 & 18.4 & 40.6 & 50.6 \\
SBU & 36.8 & 57.6 & 39.4 & 18.9 & 40.8 & 50.3 \\
MTGAN (Ours) & 37.2 & 57.7 & 40.2 & 19.9 & 41.2 & 51.2 \\
\hline
\end{tabular}

posal. For fair comparison with our method, we change our discriminator network into the structure of plain $\mathrm{R}$ $\mathrm{CNN}$, i.e. we remove the adversarial branch, retain the classification and regression branches, and feed the resized proposals $($ e.g. $224 \times 224)$ to the networks. On the small subset, as shown in Table 3 ( $2^{\text {nd }}$ vs. $3^{\text {rd }}$ rows), we outperform the plain R-CNN by a large margin (i.e. $19.9 \%$ vs. $18.8 \%$ ). As mentioned above, the reason is that the re-sized method might generate artifacts and the generated images are usually blurred for the those small input patches. Moreover, the performance of medium and large subsets are also worse than ours, and even worse than the performance of the baseline detector. We argue that the reason is that the resolution of input images in plain R-CCN (i.e. $224 \times 224$ ) is larger than our method (i.e. $64 \times 64)$, thus the batch size will be reduced due to the limited GPU memory when training the networks, which will degrade the detection performance.

Influence of the Generator in the MTGAN framework. To further validate the effectiveness of our proposed generator in the MTGAN framework, we design an experiment where the MTGAN generator is replaced with a simple bi-linear up-sampler. We upsample the training ROIs 4 times (i.e. to $64 \times 64$ ) and train a detector with both classification and regression branches (similar to the MTGAN discriminator), which is term as SBU in the comparison experiments. As shown in Table 4 ( $2^{\text {nd }}$ and $3^{\text {rd }}$ rows), the performance of SBU is far worse than our MTGAN method on the small subset (i.e. $18.9 \%$ vs. $19.9 \%$ ), and the reason is that the region images generated by the bi-linear up-sampling method are blurred and has many artificial traces as shown in the first column of each image group in Figure 3, which is unfriendly for classification and localization. In contrast, the region images gener- ated by our MTGAN framework are clear and some fine details are recovered (as shown in the third column of each image group in Figure 3), which is helpful for detecting small objects.

Influence of the regression branch. As shown in Figure 1, imperfect localization is one of the main sources of detection error. This is especially the case for small sized objects, where small shifts in their bounding boxes lead to failed detection results when the standard strict evaluation criteria are used (e.g. $\mathrm{AP}_{75}$ in $\mathrm{COCO}$ datasets). The regression branch in the discriminator can further refine bounding boxes and lead to more accurate localization. From Table 5 ( $1^{\text {st }}$ and $5^{\text {th }}$ row), we can observe that the AP performance on the small object subset improves by $0.9 \%$ when the regression branch is added, thus, demonstrating its effectiveness on the proposed detection pipeline. Interestingly, the detection performance on the large and medium subsets also improve slightly ( $0.2 \%$ and $0.3 \%$ respectively), since inaccurate localization also occurs in these subsets. We visualize the detection results, as shown in Figure 7, and find that our method can detect some small objects successfully, which are failed in the baseline detector. Also, our method has a more accurate location than the baseline detector in some cases of Large and Medium subsets.

Influence of the adversarial loss. To validate the effectiveness of back-propagating the adversarial loss to the generator network, we conduct an ablation experiment with/without this loss in the objective loss function of the generator network. Table 5 (the $2^{\text {nd }}$ and $5^{\text {th }}$ row) shows that the AP performance on the small subset drops by $0.5 \%$ without the adversarial loss. The reason is that the generated images without adversarial loss are over-smooth and lack high frequency information, which is important for object detection. Upon 
Table 5 Performance of our MTGAN model trained with and without the regression branch, adversarial loss, classification loss, and regression loss on the COCO minival subset. "reg+" indicates the regression branch in the discriminator, "adv" denotes the adversarial loss in Eq (5), "cls" represents the classification loss in Eq (6), and "reg" indicates the regression loss in $\mathrm{Eq}(7)$.

\begin{tabular}{r|lll|lll}
\hline Methods & $\mathrm{AP}$ & $\mathrm{AP}_{50}$ & $\mathrm{AP}_{75}$ & $\mathrm{AP}_{S}$ & $\mathrm{AP}_{M}$ & $\mathrm{AP}_{L}$ \\
\hline \hline w/o reg+ branch & 36.7 & 57.5 & 39.8 & 19.0 & 40.9 & 49.9 \\
w/o adv loss & 37.0 & 57.6 & 40.0 & 19.4 & 41.0 & 51.0 \\
w/o cls loss & 36.8 & 57.6 & 39.9 & 19.2 & 41.1 & 50.3 \\
w/o reg loss & 36.7 & 57.6 & 39.7 & 19.1 & 41.1 & 50.2 \\
MTGAN (Ours) & $\mathbf{3 7 . 2}$ & $\mathbf{5 7 . 7}$ & $\mathbf{4 0 . 2}$ & $\mathbf{1 9 . 9}$ & $\mathbf{4 1 . 2}$ & $\mathbf{5 1 . 2}$ \\
\hline
\end{tabular}

Table 6 Comparison of our proposed method MTGAN with the ensemble model (i.e. MTGAN + baseline detector) on the COCO minival subset.

\begin{tabular}{r|l|lll|lll}
\hline Methods & Backbone & $\mathrm{AP}$ & $\mathrm{AP}_{50}$ & $\mathrm{AP}_{75}$ & $\mathrm{AP}_{S}$ & $\mathrm{AP}_{M}$ & $\mathrm{AP}_{L}$ \\
\hline \hline Faster-RCNN (Baseline) & ResNet-50-C4 & 36.5 & 57.3 & 39.3 & 18.4 & 40.6 & 50.6 \\
MTGAN (Ours) & ResNet-50 & 37.2 & 57.7 & 40.2 & 19.9 & 41.2 & 51.2 \\
Ensemble & ResNet-50-C4 + ResNet-50 & 38.1 & 58.5 & 40.9 & 20.8 & 42.0 & 51.9 \\
\hline
\end{tabular}

close inspecting the generated images without the adversarial loss, we find that the fine details of the small objects are of low quality. To encourage the generator to produce high-quality images for better detection, we add the adversarial loss the to objective loss function of the generator network.

Influence of the classification loss. In order to verify the positive influence of the classification loss in the objective loss function of the generator network, an ablation experiment is designed by removing it from the generator loss. From Table 5 (the $3^{\text {rd }}$ and $5^{\text {th }}$ row), we see that the AP performance increases by about $1 \%$ on the small subset when the classification loss is incorporated. Clearly, this validates the claim that the classification loss promotes the generator to recover finer detailed information for better classification. In doing so, the discriminator can exploit the fine details to predict the correct category of the ROI images. The better performance indicates the importance of classification loss in the generator loss function. When visualizing the generated images, we find that the surface features (e.g. the fur of a cat and a sheep) of the objects are sharper when adding the classification loss. Maybe these surface features are the most important information for the discriminator to classify the object from the complex background (or similar category) when the objects are too small and heavily blurred. So we introduce the classification loss into the loss function of the generator network.

Influence of the regression loss. To validate the effectiveness of introducing the regression loss to the generator network, we also conduct an ablation experiment with and without this loss in the objective loss function of the generator network. As shown in Table 5 (the $4^{\text {th }}$ and $5^{\text {th }}$ row), the AP performance increases by nearly $1 \%$ absolutely on the small subset by using the regression loss to train the generator network. Similar to the classification loss, the regression loss drives the generator to recover some fine details for better localization. The increased AP demonstrates the necessity of introducing the regression loss to the generator loss function, therefore, we further add the regression loss to the generator loss function in our proposed MTGAN framework.

Can Integrating Results from Two Different Stages Improve the Performance? As done in the previous works (Cheng et al., 2018b,a; Zhang et al., 2019c,e; Shen et al., 2019), integrating results of different models can further improve the performance. So there is a question that can integrating results from two different stages in our method improve the detection performance? To answer this question, we conduct an experiment to verify if integrating results from two different stages can further improve the performance. Specifically, as done in (Cheng et al., 2018b), region scores (i.e. classification score) of our MTGAN framework is aggregated with region scores of our baseline detector by element-wise product to form the final classification score of each region. As show in Table 6, the ensemble model can indeed achieve the better performance, and the AP performance on each subset increased by abount $1.0 \%$ absolutely. However, the baseline detector and our MTGAN framework are trained separately and the final scores are combined during the testing procedure, that is to say the training and testing processes are not done in an end-to-end manner, which greatly reduces the efficiency. In the future, we plane to integrate results of our MTGAN framework and the baseline detector in an end-to-end manner as 
Table 7 The performance (AP) of the proposed MTGAN framework and other state-of-the-art methods on COCO test - dev subset. "+++" denotes the more complex training/test stages, which includes multi-scale train/test, horizontal flip train/test and OHEM (Shrivastava et al., 2016a) in the Faster RCNN.

\begin{tabular}{|c|c|c|c|c|c|c|c|}
\hline Methods & Backbone & AP & $\mathrm{AP}_{50}$ & $\mathrm{AP}_{75}$ & $\mathrm{AP}_{S}$ & $\mathrm{AP}_{M}$ & $\mathrm{AP}_{L}$ \\
\hline SSD512 (Liu et al., 2016) & VGG16 & 26.8 & 46.5 & 27.8 & 9.0 & 28.9 & 41.9 \\
\hline YOLO9000 (Redmon and Farhadi, 2017b) & Darknet-19 & 21.6 & 44.0 & 19.2 & 5.0 & 22.4 & 35.5 \\
\hline Faster RCNN+++ (He et al., 2016) & ResNet-101-C4 & 34.9 & 55.7 & 37.4 & 15.6 & 38.7 & 50.9 \\
\hline FPN (Lin et al., 2017) & ResNet-101-FPN & 36.2 & 59.1 & 39.0 & 18.2 & 39.0 & 48.2 \\
\hline G-RMI (Huang et al., 2017) & Inception-ResNet-v2 & 34.7 & 55.5 & 36.7 & 13.5 & 38.1 & 52.0 \\
\hline TDM (Shrivastava et al., 2016b) & Inception-ResNet-v2-TDM & 36.8 & 57.7 & 39.7 & 16.2 & 39.8 & 52.1 \\
\hline Mask RCNN (He et al., 2017) & ResNeXt-101-FPN & 39.8 & 62.3 & 43.4 & 22.1 & 43.2 & 51.2 \\
\hline MTGAN (Ours) & ResNet-101 & 41.4 & 63.2 & 45.4 & 24.7 & 44.2 & 52.6 \\
\hline
\end{tabular}

done in (Cheng et al., 2018a), but this is out scope of this paper.

\subsection{State-of-the-Art Comparison}

In this sub-section, we compare our proposed method with state-of-the-art methods on two challenging object detection benchmark, i.e. COCO (Lin et al., 2014) dataset and WIDER FACE (Yang et al., 2016) dataset.

\subsubsection{Evaluation on COCO Datatset}

We compare our proposed method (MTGAN) with several state-of-the-art object detectors (Liu et al., 2016; Redmon and Farhadi, 2017b; He et al., 2016; Lin et al., 2017; Huang et al., 2017; Shrivastava et al., 2016b; He et al., 2017) on the COCO test - dev subset. Table 7 lists the performance of every detector, from which we conclude that our method surpasses all other state-ofthe-art methods on all subsets. More importantly, our MTGAN achieves the highest performance (24.7\%) on the small subset, outperforming the second best object detector by about $3 \%$ absolutely. This AP improvement is the most notable for the small object subset, which clearly demonstrates the effectiveness of our method on small object detection.

Compared to these state-of-the-art CNN-based methods, the boosting of our performance mainly comes from three contributions: (1) our up-sampling network in the generator learns a clear super-resolution image, which reduces too much information loss caused by down-sampling while implementing convolution operations on small objects. Based on the super-resolution images, it is easier for the discriminator to classify the objects from the background or from the similar object categories than depending on the low-resolution blurred images; (2) the regression branch in the discriminator network further refines the location of the candidate objects cropped from the baseline detector, as a result, our method can correctly detect the object candidates whose bounding-boxes are inaccurate; (3) the classification loss $\mathrm{Eq}(6)$ promotes the generator network to learn the clear surface feature for easier classification. Meanwhile, the regression loss $\mathrm{Eq}(7)$ further encourages the generator to recover a clearer object contour for easier regression. It is not surprising that such improvement in AP performance is achieved on the clear and sharp generated super-resolution images.

Furthermore, we also achieve the highest performance $(44.2 \% / 52.6 \%)$ on the large/medium subsets, outperforming the previous state-of-the-art object detector by $1.0 \%$ and $0.5 \%$ respectively. This is because the bounding-boxes of some big objects are inaccurate, which fails to fulfill the standard evaluation criteria of COCO dataset (i.e. IoU > [0.5:0.05:0.95]). However, our method can alleviate this problem and detect these objects successfully.

\subsubsection{Evaluation on WIDER FACE Dataset}

To further validate the robustness of our proposed MTGAN framework, we conduct the experiments on the WIDER FACE dataset. Table 8 shows the AP performance of our method against the some previous stateof-the-art face detectors (Yang et al., 2016; Zhang et al., 2016; Zhu et al., 2017b; Hu and Ramanan, 2017b; Najibi et al., 2017; Zhang et al., 2017b; Wang et al., 2017b,a; Zhu et al., 2018; Zhang et al., 2018a) on WIDER FACE validation set. From Table 8, we can observe that the AP performance of our proposed method (MTGAN) surpasses the baseline face detector (SRFACE) by $2 \%$ absolutely on the Hard subset. Moreover, our method achieves the best performance (i.e. 89.3\%) on the Hard subset when compared with the previous state-of-theart face detectors, and the AP performance outperforms the second best face detector by $0.5 \%$ absolutely. Interestingly, we also get the highest AP performance $(95.9 \% / 94.8 \%)$ on the Easy/Medium subsets, surpassing the state-of-the-art face detectors by $0.6 \%$ and $0.6 \%$ respectively. 
Table 8 The detection performance (AP) of our proposed MTGAN framework and the other state-of-the-art face detectors on the WIDER FACE validation set.

\begin{tabular}{r|ccc}
\hline Method & Easy & Medium & Hard \\
\hline \hline MSCNN(Yang et al., 2016) & 0.691 & 0.64 & 0.424 \\
MTTCNN(Zhang et al., 2016) & 0.848 & 0.825 & 0.598 \\
CMS-RCNN(Zhu et al., 2017b) & 0.899 & 0.874 & 0.624 \\
HR(Hu and Ramanan, 2017b) & 0.925 & 0.910 & 0.806 \\
SSH(Najibi et al., 2017) & 0.931 & 0.921 & 0.845 \\
SFD(Zhang et al., 2017b) & 0.937 & 0.924 & 0.852 \\
Face R-FCN(Wang et al., 2017b) & 0.947 & 0.935 & 0.847 \\
FAN(Wang et al., 2017a) & 0.953 & 0.942 & 0.888 \\
Zhu et. al (Zhu et al., 2018) & 0.949 & 0.935 & 0.865 \\
FDNet(Zhang et al., 2018a) & 0.953 & 0.942 & 0.888 \\
\hline \hline SRFACE (Baseline)(Bai et al., 2018a) & 0.944 & 0.933 & 0.873 \\
MTGAN (Ours) & $\mathbf{0 . 9 5 9}$ & $\mathbf{0 . 9 4 8}$ & $\mathbf{0 . 8 9 3}$ \\
\hline
\end{tabular}

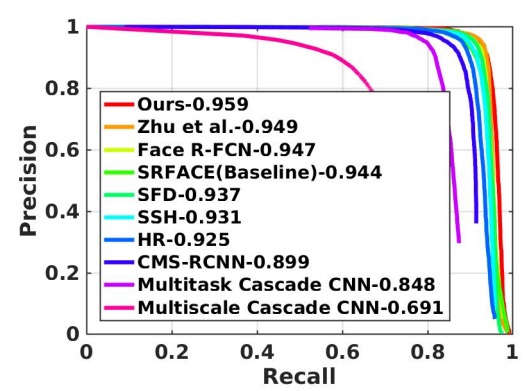

(a) Large

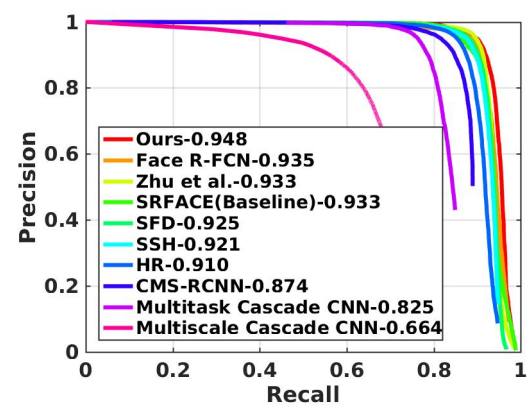

(b) Medium

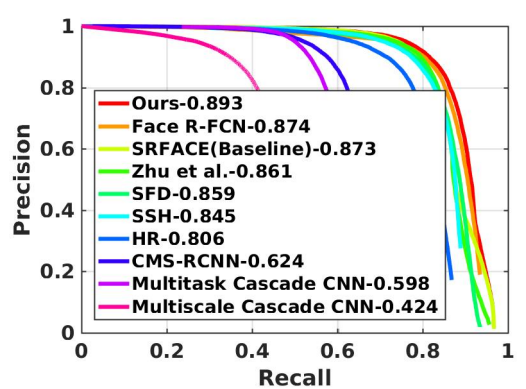

(c) Small

Fig. 4 On the WIDER FACE validation set, we compare our proposed method with our baseline method(Bai et al., 2018a) and several previous state-of-the-art methods: MSCNN(Yang et al., 2016), MTTCNN(Zhang et al., 2016), CMS-RCNN(Zhu et al., 2017b), HR(Hu and Ramanan, 2017b), SSH(Najibi et al., 2017), SFD(Zhang et al., 2017b), Face R-FCN(Wang et al., 2017b), and Zhu et. al (Zhu et al., 2018). The average precision (AP) is reported in the legend. Best viewed in color and zoomed in.

Figure 4 and 5 present the precision-recall curve of our proposed method and the previous state-of-theart methods for face detection on the WIDER FACE validation and test sets respectively. As shown in Figure 4 and 5, our method achieves superior results on this large-scale face detection dataset, especially on the Hard subset. These results further demonstrate the effectiveness of the proposed MTGAN framework for small object detection.

\subsection{Overall Error Analysis on the Achieved Performance}

After achieving the super-resolved images with rich details from the generator network, the discriminator network with multi-task losses can further distinguish the hard positive examples from the background patches (i.e. achieving lower false positives). Similarly, the discriminator network can further find some background cases in the object patches (i.e. achieving lower false negatives), which look more like objects and classify as
Table 9 The classification accuracy of the discriminator model trained with the SBU and MTGAN, where "SBU" denotes the simple bi-linear up-sampling method, "Acc." denotes the classification accuracy, "Pos." denotes the positive examples (objects), "Neg." denotes the negative examples (backgrounds), and "All" denotes all RoIs.

\begin{tabular}{l|lll}
\hline Method & Acc. of Pos. & Acc. of Neg. & Acc. of All \\
\hline \hline SBU & 0.942 & 0.963 & 0.955 \\
MTGAN(Ours) & 0.966 & 0.972 & 0.970 \\
\hline
\end{tabular}

objects in the baseline detector. To verify the above claims, we conduct an experiment to show classification accuracy of the discriminator. Specifically, we first calculate the IoU between the RoIs from our baseline detector and the ground truth bounding-boxes. If the IoU of an RoI is larger than 0.5 we mark it as a positive example (i.e. objects), and we mark it as a negative example (i.e. backgrounds) if the IoU of an RoI is less than 0.35 . Then, the positive and negative examples are inputted to the generator network and up-sampled by 


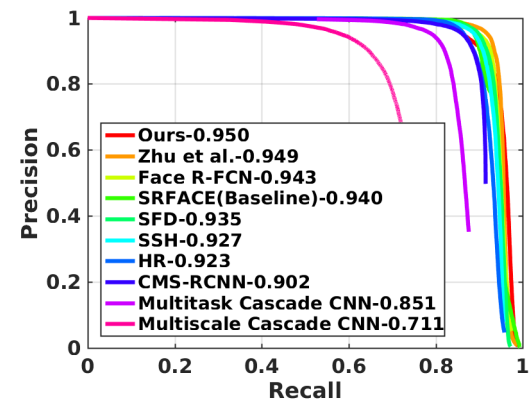

(a) Large

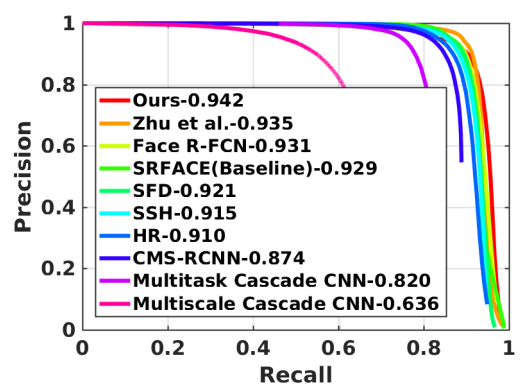

(b) Medium

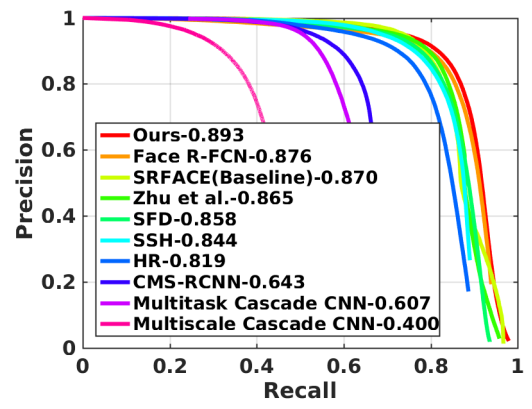

(c) Small

Fig. 5 On the WIDER FACE test set, we compare our proposed method with our baseline method(Bai et al., 2018a) and several previous state-of-the-art methods: MSCNN(Yang et al., 2016), MTTCNN(Zhang et al., 2016), CMS-RCNN(Zhu et al., 2017b), HR(Hu and Ramanan, 2017b), SSH(Najibi et al., 2017), SFD(Zhang et al., 2017b), Face R-FCN(Wang et al., 2017b), and Zhu et. al (Zhu et al., 2018). The average precision (AP) is reported in the legend. Best viewed in color and zoomed in.
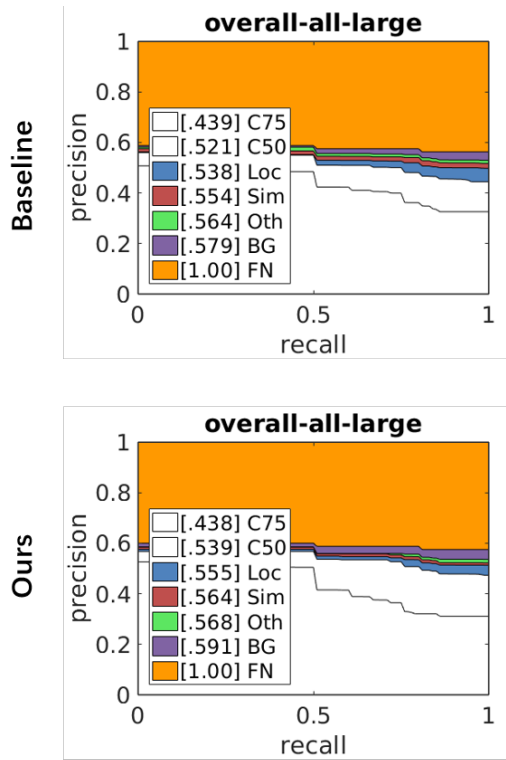

(a) Large
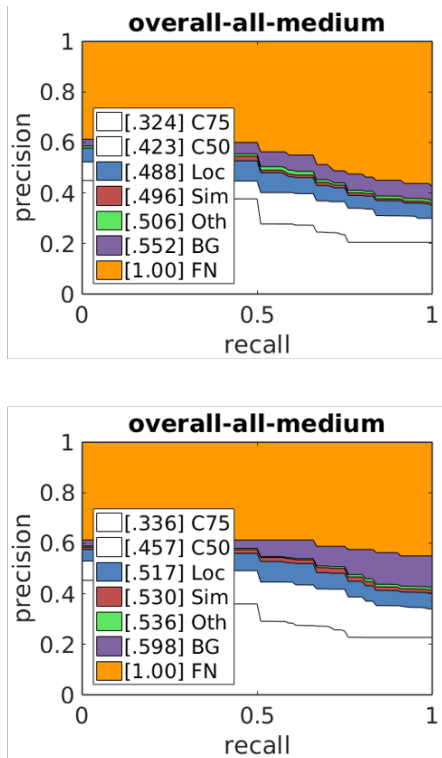

(b) Medium
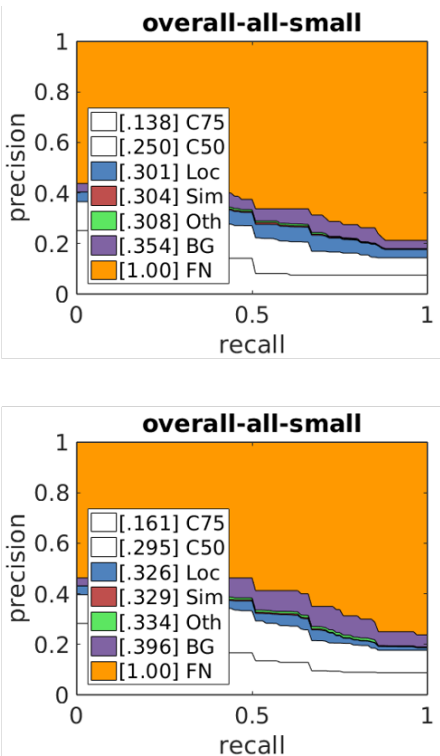

(c) Small

Fig. 6 The overall error analysis on the performance of the baseline detector and our proposed method over all categories on the large, medium and small subsets of the COCO minival subset, respectively. The plots in each sub-image are a series of precision-recall curves under different evaluation settings defined in (Lin et al., 2014). The definition of legends in each sub-image can be found on the official website of COCO dataset ${ }^{6}$. Best seen on the computer, in color and zoomed in.

a simple bi-linear up-sampler (SBU) to generated the super-resolved images, respectively. Finally, the superresolved images go through the discriminator to output the classification accuracy. As shown in Table 9, classification accuracy of our proposed MTGAN outperforms the SBU method by a large margin on positive and negative examples, which demonstrates the effectiveness of the super-resolution network.

To further analyze the error types, we show the overall error analysis for the achieved performance. As show in Figure 6, our MTGAN framework achieve higher

\footnotetext{
${ }^{6}$ http://cocodataset.org/\#detection-eval
}

performance on each case than the baseline detector. Since we focus on detecting small objects in this paper, we take the error types on the small subset (i.e. Figure 6 (c)) as an example to analyze the error types. The area under each curve in Figure 6 is shown in brackets in the legend. Under the strict evaluation metrics $\mathrm{IoU}=0.75$, overall $\mathrm{AP}$ (i.e. $\mathrm{C} 75$ ) of our proposed method on the small subset is $16.1 \%$, outperforming the baseline method by a large margin (i.e. $2.3 \%$ ), which demonstrates the effectiveness of the proposed method on detecting small objects. In the case of Loc (localization errors ignored), our proposed method improves 


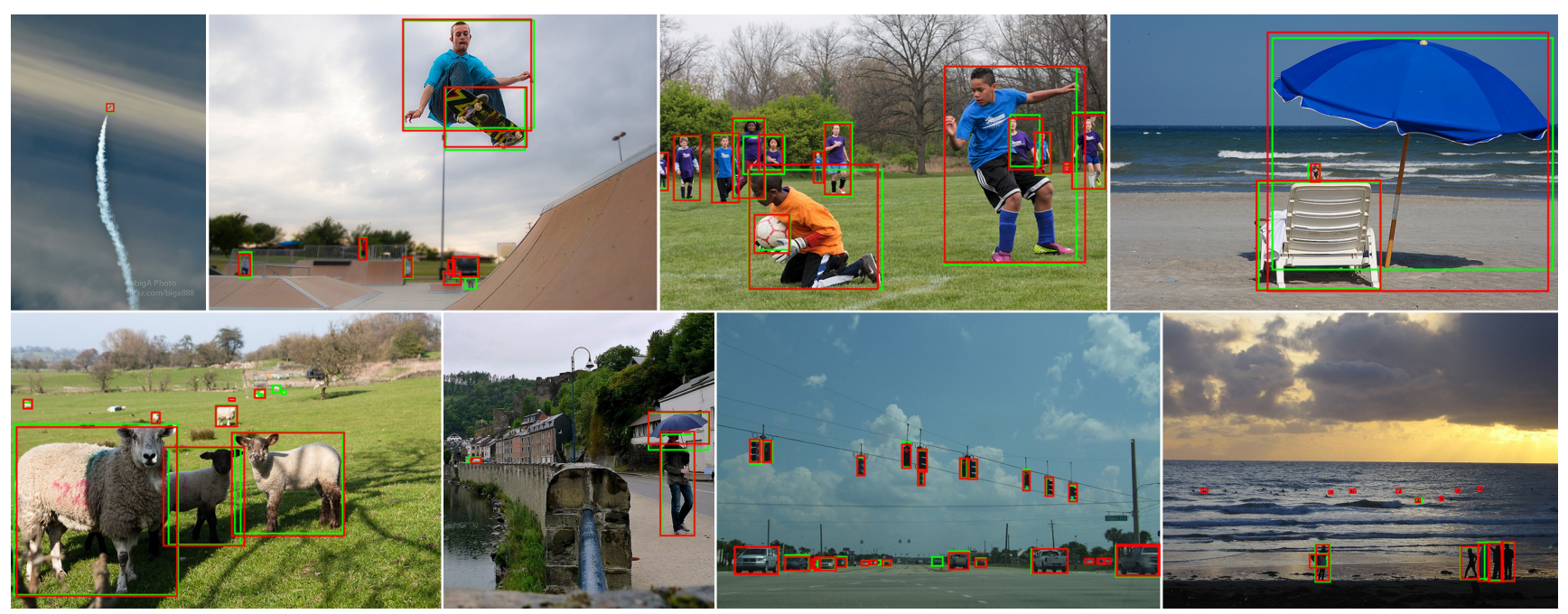

Fig. 7 Qualitative detection results of our proposed MTGAN framework on COCO dataset. Green and red boxes denote the ground-truths and the results of our method respectively. Best seen on the computer, in color and zoomed in.

the AP performance to $32.6 \%$ and we exceed the baseline method (i.e. $30.1 \%$ ) by $2.5 \%$. For the gap between Loc and C50, we are less than the baseline method $(32.6 \%-29.5 \%=3.1 \%$ vs. $30.1 \%-25.0 \%=5.1 \%)$, which means that our proposed method has a better regression ability than the baseline method. For the metric Oth, which removes all class confusions, we raise AP to $33.4 \%$ compared $30.8 \%$ in the baseline method. Interesting, after removing all background and class confusion false positives (i.e. BG), our proposed method achieves $39.6 \%$ in AP performance, surpassing the baseline method by $4.1 \%$ absolutely. The difference of the improved AP performance in BG and Oth metrics between our method and the baseline method is $1.5 \%$ (i.e. $4.1 \%-(33.4 \%-30.8 \%)=1.5 \%)$, and this improvement means the proposed method can further detect some hard positive examples from the complex backgrounds, i.e. we can recall more small objects. When calculating the gap between the BG and Loc, we are $7.0 \%$ compared to $5.3 \%$ in the baseline method. We think the reason is that our method classifies some backgrounds as the objects, and we will further explore this problem in the future. In summary, our proposed method has better classification and regression ability than the baseline method in the task of detecting small objects.

\subsection{Qualitative Results}

We show some detection results generated by the proposed MTGAN framework on COCO and WIDER FACE datasets in Figure 7 and Figure 8 respectively. From
Figure 7 and Figure 8, we observe that our method successfully finds almost all the objects, even though some ones are very small. This demonstrates the effectiveness of our detector on the small object detection problem. Figure 7 and Figure 8 also show some failure cases including some false negative and positive results. By carefully inspecting the detection results, we find that the majority of these failure cases are some backgrounds that are very similar to a small blurred object. These failure results indicate that more progress is needed to further improve the small object detection performance. We will focus on extracting robust features for these more challenging small objects in the future.

\section{Conclusion}

In this paper, we propose an end-to-end multi-task GAN (MTGAN) framework to detect small objects (i.e. including general objects and faces) in unconstrained scenarios. In the MTGAN, the generator up-samples the small blurred ROI images to fine-scale clear images. Moreover, We introduce the extra classification and regression branches into the discriminator network, which can distinguish the fake/real, classify the object category, and regress the object location simultaneously. Our generator and discriminator networks are trained end-to-end in an alternative way. To recover detailed information for better detection, the classification and regression losses in the discriminator network are further propagated back to the objective loss function of the generator network. Extensive experiments on the 


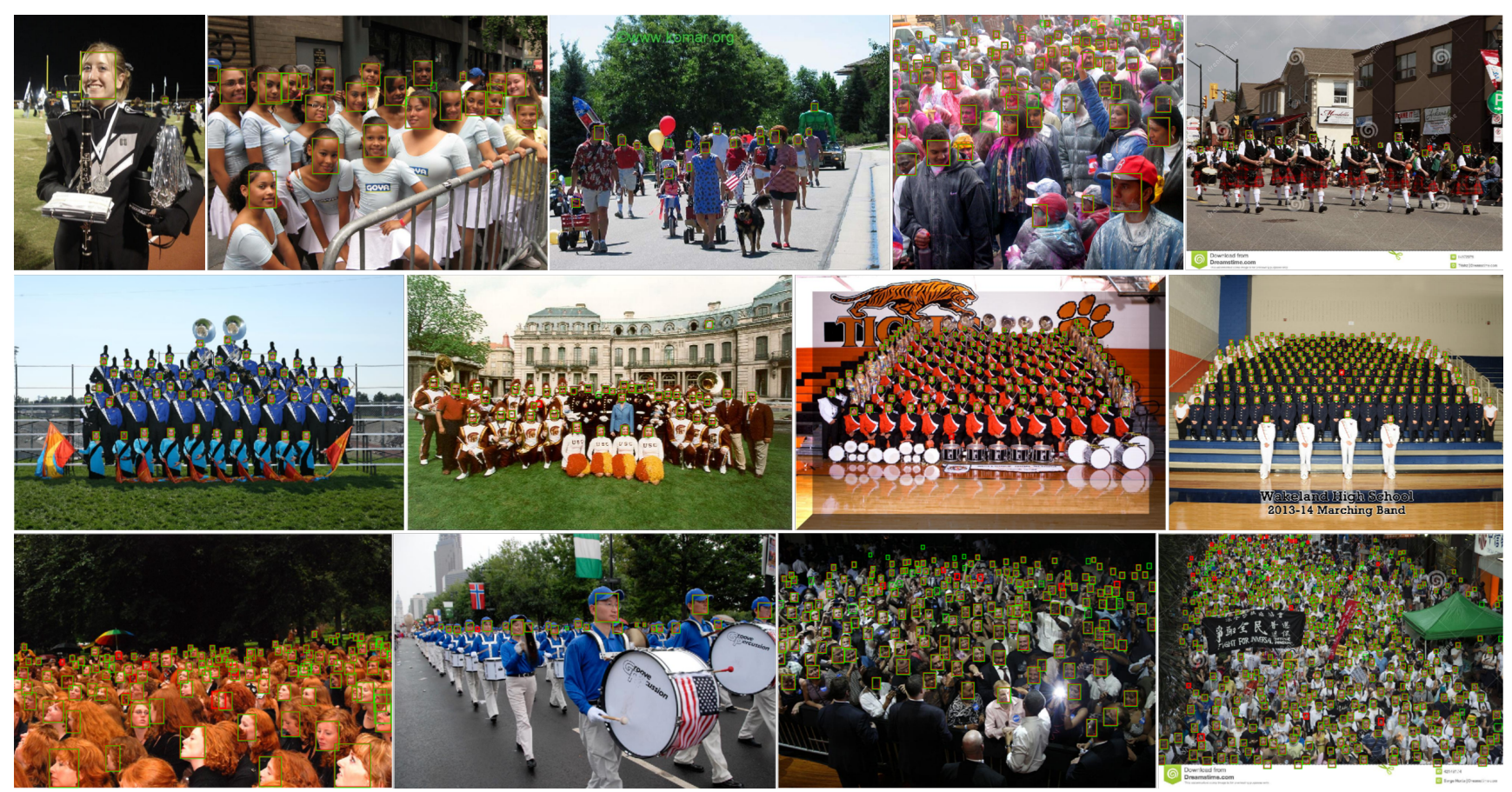

Fig. 8 Qualitative detection results of our proposed MTGAN framework on WIDER FACE dataset. Green bounding boxes are ground truth annotations and red bounding boxes are the results from our method. Best seen on the computer, in color and zoomed in.

challenging COCO and WIDER FACE datasets demonstrate that our proposed method improves state-of-theart AP performance in general, where the largest improvement is observed for small sized objects.

Acknowledgements The majority of this work was done when Yongqiang Zhang was a visiting $\mathrm{PhD}$ student at king Abdullah University of Science and Technology (KAUST), and the others are continued at Harbin Institute of Technology (HIT). This work was supported by Natural Science Foundation of China, Grant No. 61603372.

\section{References}

Bai Y, Ghanem B (2017) Multi-branch fully convolutional network for face detection. arXiv preprint arXiv:170706330 4

Bai Y, Zhang Y, Ding M, Ghanem B (2018a) Finding tiny faces in the wild with generative adversarial network. CVPR IEEE 3, 4, 6, 10, 15, 16

Bai Y, Zhang Y, Ding M, Ghanem B (2018b) Sodmtgan: Small object detection via multi-task generative adversarial network. Computer Vision-ECCV pp 8-14 3, 6

Bell S, Lawrence Zitnick C, Bala K, Girshick R (2016a) Inside-outside net: Detecting objects in context with skip pooling and recurrent neural networks. In: Pro- ceedings of the IEEE conference on computer vision and pattern recognition, pp 2874-2883 4

Bell S, Zitnick CL, Bala K, Girshick R (2016b) Insideoutside net: Detecting objects in context with skip pooling and recurrent neural networks. CVPR 9

Cai Z, Vasconcelos N (2018) Cascade r-cnn: Delving into high quality object detection. In: CVPR 3

Cai Z, Fan Q, Feris RS, Vasconcelos N (2016) A unified multi-scale deep convolutional neural network for fast object detection. In: european conference on computer vision, Springer, pp 354-370 4

Cheng B, Wei Y, Shi H, Feris R, Xiong J, Huang T (2018a) Decoupled classification refinement: Hard false positive suppression for object detection. arXiv preprint arXiv:181004002 13, 14

Cheng B, Wei Y, Shi H, Feris R, Xiong J, Huang T (2018b) Revisiting rcnn: On awakening the classification power of faster rcnn. In: Proceedings of the European Conference on Computer Vision (ECCV), pp 453-468 13

Chi C, Zhang S, Xing J, Lei Z, Li SZ, Zou X (2018) Selective refinement network for high performance face detection. arXiv preprint arXiv:180902693 4

Dai J, Li Y, He K, Sun J (2016) R-fcn: Object detection via region-based fully convolutional networks. In: NIPS, pp 379-387 1

Denton EL, Chintala S, Fergus R, et al. (2015) Deep generative image models using a laplacian pyramid 
of adversarial networks. In: Advances in neural information processing systems, pp 1486-1494 5

Dong C, Loy CC, Tang X (2016) Accelerating the super-resolution convolutional neural network. In: European conference on computer vision, Springer, pp 391-407 6

Fu CY, Liu W, Ranga A, Tyagi A, Berg AC (2017) Dssd: Deconvolutional single shot detector. arXiv preprint arXiv:170106659 4

Girshick R (2015) Fast r-cnn. In: ICCV, IEEE, pp 14401448 4, 7, 8, 10

Girshick R, Donahue J, Darrell T, Malik J (2014) Rich feature hierarchies for accurate object detection and semantic segmentation. In: CVPR, pp 580-587 3, 10, 11

Girshick R, Radosavovic I, Gkioxari G, Dollár P, He K (2018) Detectron. https://github.com/ facebookresearch/detectron 11

Goodfellow I, Pouget-Abadie J, Mirza M, Xu B, WardeFarley D, Ozair S, Courville A, Bengio Y (2014) Generative adversarial nets. In: Advances in neural information processing systems, pp 2672-2680 2, 5, 6, 7, 9, 10

He K, Zhang X, Ren S, Sun J (2015) Delving deep into rectifiers: Surpassing human-level performance on imagenet classification. In: ICCV, pp 1026-1034 6

He K, Zhang X, Ren S, Sun J (2016) Deep residual learning for image recognition. In: Proceedings of the IEEE conference on computer vision and pattern recognition, pp 770-778 5, 7, 10, 14

He K, Gkioxari G, Dollar P, Girshick R (2017) Mask r-cnn. In: CVPR, pp 2961-2969 10, 11, 14

Hradiš M, Kotera J, Zemcík P, Šroubek F (2015) Convolutional neural networks for direct text deblurring. In: Proceedings of BMVC, vol 10, p 26

$\mathrm{Hu}$ P, Ramanan D (2017a) Finding tiny faces. In: Computer Vision and Pattern Recognition (CVPR), 2017 IEEE Conference on, IEEE, pp 1522-1530 4

$\mathrm{Hu}$ P, Ramanan D (2017b) Finding tiny faces. In: CVPR, IEEE, pp 1522-1530 14, 15, 16

Huang J, Rathod V, Sun C, Zhu M, Korattikara A, Fathi A, Fischer I, Wojna Z, Song Y, Guadarrama S, et al. (2017) Speed/accuracy trade-offs for modern convolutional object detectors. In: IEEE CVPR 14

Ioffe S, Szegedy C (2015) Batch normalization: Accelerating deep network training by reducing internal covariate shift. In: ICML, pp 448-456 6

Isola P, Zhu JY, Zhou T, Efros AA (2017) Image-toimage translation with conditional adversarial networks. In: CVPR, pp 1125-1134 5, 7, 8

Jain V, Learned-Miller E (2010) Fddb: A benchmark for face detection in unconstrained settings. Tech. rep., Technical Report UM-CS-2010-009, University of Massachusetts, Amherst 4

Jiang H, Learned-Miller E (2017) Face detection with the faster r-cnn. In: Automatic Face \& Gesture Recognition (FG 2017), 2017 12th IEEE International Conference on, IEEE, pp 650-657 4

Kim J, Kwon Lee J, Mu Lee K (2016) Accurate image super-resolution using very deep convolutional networks. In: Proceedings of the IEEE conference on computer vision and pattern recognition, pp 164616546

Kingma DP, Ba J (2014) Adam: A method for stochastic optimization. arXiv preprint arXiv:14126980 10

Krizhevsky A, Sutskever I, Hinton GE (2012a) Imagenet classification with deep convolutional neural networks. In: NIPS, pp 1097-1105 3

Krizhevsky A, Sutskever I, Hinton GE (2012b) Imagenet classification with deep convolutional neural networks. In: Advances in neural information processing systems, pp 1097-1105 5

Ledig C, Theis L, Huszar F, Caballero J, Cunningham A, Acosta A, Aitken A, Tejani A, Totz J, Wang Z, et al. (2017) Photo-realistic single image superresolution using a generative adversarial network. In: CVPR, pp 4681-4690 2, 5, 6, 7, 8

Li J, Wang Y, Wang C, Tai Y, Qian J, Yang J, Wang C, Li J, Huang F (2018) Dsfd: Dual shot face detector. arXiv preprint arXiv:181010220 4

Lin TY, Maire M, Belongie S, Hays J, Perona P, Ramanan D, Dollár P, Zitnick CL (2014) Microsoft coco: Common objects in context. In: ECCV, Springer, pp 740-755 2, 3, 9, 10, 14, 16

Lin TY, Dollár P, Girshick R, He K, Hariharan B, Belongie S (2017) Feature pyramid networks for object detection. In: CVPR, vol 1, p 4 1, 2, 4, 5, 9, 10, 14

Liu W, Anguelov D, Erhan D, Szegedy C, Reed S, Fu CY, Berg AC (2016) Ssd: Single shot multibox detector. In: ECCV, Springer, pp 21-37 1, 2, 4, 5, 14

Mathieu MF, Zhao JJ, Zhao J, Ramesh A, Sprechmann P, LeCun Y (2016) Disentangling factors of variation in deep representation using adversarial training. In: Advances in Neural Information Processing Systems, pp 5040-5048 5

Najibi M, Samangouei P, Chellappa R, Davis LS (2017) Ssh: Single stage headless face detector. In: Proceedings of the IEEE International Conference on Computer Vision, pp 4875-4884 4, 14, 15, 16

Radford A, Metz L, Chintala S (2015) Unsupervised representation learning with deep convolutional generative adversarial networks. arXiv preprint arXiv:151106434 7

Redmon J, Farhadi A (2017a) Yolo9000: Better, faster, stronger. 2017 IEEE Conference on Computer Vision 
and Pattern Recognition (CVPR) pp 6517-6525 4

Redmon J, Farhadi A (2017b) Yolo9000: Better, faster, stronger. In: CVPR, IEEE, pp 6517-6525 14

Redmon J, Divvala S, Girshick R, Farhadi A (2016a) You only look once: Unified, real-time object detection. In: CVPR, pp 779-788 1

Redmon J, Divvala S, Girshick R, Farhadi A (2016b) You only look once: Unified, real-time object detection. In: Proceedings of the IEEE conference on computer vision and pattern recognition, pp 779-788 4

Ren S, He K, Girshick R, Sun J (2015) Faster r-cnn: Towards real-time object detection with region proposal networks. In: NIPS, pp 91-99 1, 4, 5, 10, 11

Shen Z, He Z, Xue X (2019) Meal: Multi-model ensemble via adversarial learning. In: AAAI 13

Shiri F, Yu X, Porikli F, Hartley R, Koniusz P (2019) Identity-preserving face recovery from stylized portraits. International Journal of Computer Vision pp $1-215$

Shrivastava A, Gupta A, Girshick R (2016a) Training region-based object detectors with online hard example mining. In: CVPR, pp 761-769 14

Shrivastava A, Sukthankar R, Malik J, Gupta A (2016b) Beyond skip connections: Top-down modulation for object detection. CoRR abs/1612.06851 14

Simonyan K, Zisserman A (2014) Very deep convolutional networks for large-scale image recognition. arXiv preprint arXiv:14091556 3, 5

Song Y, Zhang J, Gong L, He S, Bao L, Pan J, Yang Q, Yang MH (2019) Joint face hallucination and deblurring via structure generation and detail enhancement. International Journal of Computer Vision 127(6-7):785-800 5

Tang X, Du DK, He Z, Liu J (2018) Pyramidbox: A context-assisted single shot face detector. arXiv preprint arXiv:180307737 4

Viola P, Jones MJ (2004) Robust real-time face detection. International journal of computer vision 57(2):137-154 4

Wan S, Chen Z, Zhang T, Zhang B, Wong Kk (2016) Bootstrapping face detection with hard negative examples. arXiv preprint arXiv:160802236 4

Wang J, Yuan Y, Yu G (2017a) Face attention network: An effective face detector for the occluded faces. arXiv preprint arXiv:171107246 4, 14, 15

Wang Y, Ji X, Zhou Z, Wang H, Li Z (2017b) Detecting faces using region-based fully convolutional networks. arXiv preprint arXiv:170905256 14, 15, 16

Wang Z, Liu D, Yang J, Han W, Huang T (2015) Deep networks for image super-resolution with sparse prior. In: Proceedings of the IEEE international conference on computer vision, pp 370-378 6
Wen Y, Zhang K, Li Z, Qiao Y (2019) A comprehensive study on center loss for deep face recognition. International Journal of Computer Vision pp 1-16 4

Yan J, Zhang X, Lei Z, Li SZ (2013) Real-time high performance deformable model for face detection in the wild. In: Biometrics (ICB), 2013 International Conference on, Citeseer, pp 1-6 4

Yan J, Lei Z, Wen L, Li SZ (2014) The fastest deformable part model for object detection. In: Proceedings of the IEEE Conference on Computer Vision and Pattern Recognition, pp 2497-2504 4

Yang S, Luo P, Loy CC, Tang X (2016) Wider face: A face detection benchmark. In: Proceedings of the IEEE conference on computer vision and pattern recognition, pp 5525-5533 3, 4, 9, 10, 14, 15, 16

Zhang C, Xu X, Tu D (2018a) Face detection using improved faster rcnn. arXiv preprint arXiv:180202142 14,15

Zhang H, Riggan BS, Hu S, Short NJ, Patel VM (2019a) Synthesis of high-quality visible faces from polarimetric thermal faces using generative adversarial networks. International Journal of Computer Vision 127(6-7):845-862 5

Zhang J, Wu X, Zhu J, Hoi SC (2017a) Feature agglomeration networks for single stage face detection. arXiv preprint arXiv:171200721 4

Zhang K, Zhang Z, Li Z, Qiao Y (2016) Joint face detection and alignment using multitask cascaded convolutional networks. IEEE Signal Processing Letters 23(10):1499-1503 14, 15, 16

Zhang S, Zhu X, Lei Z, Shi H, Wang X, Li SZ (2017b) S3fd: Single shot scale-invariant face detector. In: Proceedings of the IEEE International Conference on Computer Vision, pp 192-201 14, 15, 16

Zhang S, Wen L, Shi H, Lei Z, Lyu S, Li SZ (2019b) Single-shot scale-aware network for real-time face detection. International Journal of Computer Vision pp $1-234$

Zhang Y, Ding M, Fu W, Li Y (2017c) Reading recognition of pointer meter based on pattern recognition and dynamic three-points on a line. In: ICMV 2016, International Society for Optics and Photonics, vol 10341, p 103410K 4

Zhang Y, Bai Y, Ding M, Li Y, Ghanem B (2018b) W2f: A weakly-supervised to fully-supervised framework for object detection. In: Proceedings of the IEEE Conference on Computer Vision and Pattern Recognition, pp 928-936 3

Zhang Y, Bai Y, Ding M, Li Y, Ghanem B (2018c) Weakly-supervised object detection via mining pseudo ground truth bounding-boxes. Pattern Recognition 84:68-81 3 
Zhang Y, Ding M, Bai Y, Ghanem B (2019c) Detecting small faces in the wild based on generative adversarial network and contextual information. Pattern Recognition 13

Zhang Y, Ding M, Bai Y, Liu D, Ghanem B (2019d) Learning a strong detector for action localization in videos. Pattern Recognition Letters 128:407-413 3

Zhang Y, Ding M, Bai Y, Xu M, Ghanem B (2019e) Beyond weakly-supervised: Pseudo ground truths mining for missing bounding-boxes object detection. IEEE Transactions on Circuits and Systems for Video Technology 13

Zhou B, Lapedriza A, Xiao J, Torralba A, Oliva A (2014) Learning deep features for scene recognition using places database. In: NIPS, pp 487-495 3

Zhu C, Zheng Y, Luu K, Savvides M (2017a) Cms-rcnn: contextual multi-scale region-based cnn for unconstrained face detection. In: Deep Learning for Biometrics, Springer, pp 57-79 4

Zhu C, Zheng Y, Luu K, Savvides M (2017b) Cms-rcnn: contextual multi-scale region-based cnn for unconstrained face detection. In: Deep Learning for Biometrics, Springer, pp 57-79 14, 15, 16

Zhu C, Tao R, Luu K, Savvides M (2018) Seeing small faces from robust anchors perspective. In: Proceedings of the IEEE Conference on Computer Vision and Pattern Recognition, pp 5127-5136 14, 15, 16

Zhu JY, Krähenbühl P, Shechtman E, Efros AA (2016) Generative visual manipulation on the natural image manifold. In: European Conference on Computer Vision, Springer, pp 597-613 5 